\title{
Maturation of Brain Microstructure and Metabolism Associates with Increased Capacity for Self-Regulation during the Transition from Childhood to Adolescence
}

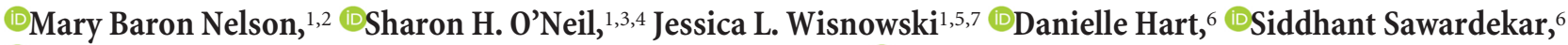 \\ Virginia Rauh, ${ }^{9,10}$ Frederica Perera, ${ }^{9,11}$ Howard F. Andrews, ${ }^{12}$ @Lori A. Hoepner, ${ }^{11,13}$ Wanda Garcia, ${ }^{9,10}$ \\ Molly Algermissen, ${ }^{14}$ Ravi Bansal, ${ }^{1,6}$ and Bradley S. Peterson ${ }^{1,4,6,8}$ \\ ${ }^{1}$ Department of Pediatrics, Keck School of Medicine at USC, Los Angeles, California 90027, ${ }^{2}$ Division of Cancer \& Blood Diseases, ${ }^{3}$ Division of \\ Neurology, ${ }^{4}$ The Saban Research Institute, ${ }^{5}$ Department of Radiology, ${ }^{6}$ Institute for the Developing Mind, and 7 Division of Neonatology, Children's \\ Hospital Los Angeles, Los Angeles, California 90027, ${ }^{8}$ Department of Psychiatry, Keck School of Medicine at University of Southern California, Los \\ Angeles, California 90027, ${ }^{9}$ Columbia Center for Children's Environmental Health, New York, New York 10025, Departments of ${ }^{10} \mathrm{Population}$ and \\ Family Health, and ${ }^{11}$ Environmental Health Sciences, and ${ }^{12}$ Biostatistics, Mailman School of Public Health, Columbia University, New York, New \\ York 10025, ${ }^{13}$ Department of Environmental and Occupational Health Sciences, SUNY Downstate School of Public Health, Brooklyn, New York \\ 11203, and ${ }^{14}$ Department of Psychiatry, Columbia University, New York, New York 10025
}

Children ages 9-12 years face increasing social and academic expectations that require mastery of their thoughts, emotions, and behavior. Little is known about the development of neural pathways integral to these improving capacities during the transition from childhood to adolescence. Among 234 healthy, inner-city male and female youth (species Homo sapiens) 9-12 years of age followed by the Columbia Center for Children's Environmental Health, we acquired diffusion tensor imaging, multiplanar chemical shift imaging, and cognitive measures requiring self-regulation. We found that increasing age was associated with increased fractional anisotropy and decreased apparent diffusion coefficient, most prominently in the frontal and cingulate cortices, striatum, thalamus, deep white matter, and cerebellum. Additionally, we found increasing age was associated with increased $N$-acetylL-aspartate (NAA) in the anterior cingulate and insular cortices, and decreased NAA in posterior cingulate and parietal cortices. Age-associated changes in microstructure and neurometabolite concentrations partially mediated age-related improvements in performance on executive function tests. Together, these findings suggest that maturation of key regions within cortico-striatalthalamo-cortical circuits subserve the emergence of improved self-regulatory capacities during the transition from childhood to adolescence.

Key words: childhood; development; imaging; maturation; self-regulation

Significance Statement

Few imaging studies of normal brain development have focused on a population of inner-city, racial/ethnic minority youth during the transition from childhood to adolescence, a period when self-regulatory capacities rapidly improve. We used DTI and MPCSI to provide unique windows into brain maturation during this developmental epoch, assessing its mediating influences on age-related improvement in performance on self-regulatory tasks. Our findings suggest that rapid maturation of cortico-striato-thalamo-cortical circuits, represented as progressive white-matter maturation (increasing FA and increasing NAA, Ch, $\mathrm{Cr}$ concentrations accompanying advancing age) in frontal regions and related subcortical projections and synaptic pruning (decreasing $\mathrm{NAA}, \mathrm{Ch}, \mathrm{Cr}, \mathrm{Glx}$ ) in posterior regions, support age-related improvements in executive functioning and self-regulatory capacities in youth 9-12 years of age. 


\section{Introduction}

The transition from childhood to adolescence is a time of emergence and strengthening of the ability to regulate thought, emotion, and behavior in the context of increasing societal demands on intellectual ability, academic performance, and interpersonal relationships. Self-regulation-monitoring and controlling one's own thoughts, emotions, and actions-supports flexible, goaldirected behavior. It is critical to academic and social competence (Nigg, 2017), and to maintaining emotional health. The neural circuits that support self-regulation include gray matter of the frontal cortex, basal ganglia, and thalamus, gray matter structures interconnected via white matter axonal pathways projecting through the centrum semiovale, corona radiata, and internal capsule. These frontostriatal circuits are a component of the larger cortico-striato-thalamo-cortical (CSTC) loops (Marsh et al., 2009a) that include dorsolateral prefrontal cortex (DLPFC), anterior cingulate cortex, supplementary motor area, ventral and lateral orbitofrontal cortex, and temporal cortices, all of which have connections with the basal ganglia and thalamus (Peterson et al., 1999, 2002; Leung et al., 2000). The dorsal portions of these circuits subserve neuropsychological tasks requiring response inhibition, or the ability to supersede prepotent responses with responses designated instead by task instructions, which is a form of self-regulation (Peterson et al., 2003; Marsh et al., 2009a). Response inhibition strengthens during late childhood and adolescence and is thought to depend on the dorsolateral and ventrolateral prefrontal cortices and their connections to the basal ganglia (Dubin et al., 2010).

Despite abundant evidence that dysfunction in these circuits underlies a wide range of developmental psychopathologies, including attention-deficit/hyperactivity disorder (Pavuluri et al., 2009; Rubia, 2011), tic disorders (Marsh et al., 2007), obsessivecompulsive disorder (Goncalves et al., 2016), addictions (Volkow et al., 2016), and eating disorders (Marsh et al., 2009b; JáureguiLobera, 2011; Kessler et al., 2016), relatively little is known about the cellular and microstructural changes in these circuits that support the maturation of self-regulatory skills in the transition from childhood to adolescence.

The aim of this study was to identify the age correlates of cerebral microstructure and neurometabolite concentrations in a large cohort of typically developing, racial and ethnic minority youth, 9-12 years of age. This is the time in development when children enter middle school and mobilize self-regulatory capacities to meet rapidly increasing academic and social demands. We also aimed to assess whether these brain measures mediate the association of advancing age with better performance on cognitive tasks requiring self-regulation.

We elected to use diffusion tensor imaging (DTI) and multiplanar chemical shift imaging (MPCSI) to measure brain maturation in CSTC pathways. DTI characterizes the three-dimensional diffusion of water as a function of spatial location, providing information about

Received Sept. 19, 2018; revised July 30, 2019; accepted Aug. 5, 2019

Author contributions: V.R., F.P., H.F.A., L.A.H., M.A., R.B., and B.S.P. designed research; V.R., F.P., L.A.H., W.G., M.A., and B.S.P. performed research; M.B.N., D.H., S.S., H.F.A., R.B., and B.S.P. analyzed data; M.B.N., S.H.O., J.L.W., V.R., F.P., R.B., and B.S.P. wrote the paper.

This work was supported by the New York State Psychiatric Institute and Columbia University, and with funding from 2 P50 ES009600 [National Institute of Environmental Health Sciences (NIEHS)], EPA 83615401, 1R01DA027100 (National Institute on Drug Abuse), 5R01ES015579 (NIEHS). We thank the Neuropsychology Core at The Saban Research Institute of Children's Hospital Los Angeles for its support.

The authors declare no competing financial interests.

Correspondence should be addressed to Bradley Peterson at bpeterson@chla.usc.edu.

https://doi.org/10.1523/JNEUROSCI.2422-18.2019

Copyright $(2019$ the authors developing white matter organization, integrity, and myelination. MPCSI, a relatively recent advance in MR spectroscopy (MRS) methodology, provides a metabolic map of brain metabolites in $1 \mathrm{cc}$ voxels throughout the brain, with a degree of spatial resolution not possible using more conventional, single-voxel MRS. Combining these two modalities permits a deeper exploration of brain tissue organization and cellular characterization of CSTC pathways in the late grade school-age child. Self-regulation was assessed using measures of performance on standardized psychometric tasks requiring attention, response inhibition, and cognitive flexibility. Additionally, we assessed visual-motor integration, because it requires similarly complex circuits to integrate visual, motor, and planning components of the task. We conducted post hoc mediation analyses to test whether brain measures mediated age-related improvement in psychometric performance.

Our a priori hypotheses were that (1) age would correlate significantly with measures of cerebral microstructure [fractional anisotropy (FA), apparent diffusion coefficient (ADC)] and neurometabolite concentrations $[N$-acetyl-L-aspartate $(\mathrm{NAA})]$ in CSTC pathways, representing progressive brain maturation from ages 9 to 12; and (2) FA and NAA measures within CSTC pathways would mediate the age-related improvement in performance on executive function tests, indicating that maturation of CSTC pathways support an increasing capacity for selfregulation during this period of development.

\section{Materials and Methods}

\section{Experimental design}

This was a cross-sectional neuroimaging and psychometric study of children ages 9-12 years who underwent assessment as part of a larger prospective, longitudinal study described in the next section.

\section{Sampling frame}

This study population of 9 to 12 year-old youth was selected from a birth cohort of the Columbia Center for Children's Environmental Health. The cohort comprised 727 children born to urban, non-smoking mothers 18-35 years of age who were without diabetes, hypertension, and HIV. Pregnant women were recruited from 1998 to 2006 through prenatal clinics as part of a prospective, longitudinal study of environmental exposures in a representative sample of low-income, African-American and Latino women in northern Manhattan. By 2014, 204 participants had withdrawn from the study or had not been recently assessed, leaving 523 participants. The demographics (age, sex, race/ethnicity, maternal age, or education) of children who discontinued participation did not differ significantly from those who continued in the study. From this sample of 523 participants, we were able to obtain MRI scans in 309 of the children, 5-14 years of age. Our primary analyses focused on the 280 children who were 9-12 years of age, a time when intellectual and selfregulatory capacities develop rapidly. Restricting the age range to 9-12 years also provided a much more uniform age distribution in the sample (Fig. 1), supporting more robust statistical analyses and inferences about the age-related effects of brain maturation during the transition from childhood to adolescence. In addition, we conducted post hoc tests across the entire cohort from 5 to 14 years of age to determine whether the age correlations in brain microstructure and metabolite concentrations generalized to the wider age range from childhood to adolescence.

Of the 319 participants in the more restricted age range, 280 had imaging data obtained within $10 \mathrm{~d}$ of psychometric assessment; 168 children had both DTI and MPCSI data, 66 had usable DTI but no MPCSI data, and 46 had MPCSI data but no usable DTI data. Thus, the final DTI sample was $234(66+168)$ and consisted of 128 females and 106 males, and the MPCSI sample was $218(46+168)$, consisting of 116 females and 102 males. Fewer children had spectroscopy data, as MPCSI was the last sequence of the scanning protocol; thus, if children were unable to complete the entire scan, spectroscopy was not obtained. Additionally, 29 
participants aged 5-8 and 13-14 had imaging and psychometric data available for post hoc analyses.

\section{Participant characterization and study procedures}

We obtained written informed consent from parents and assent from children. The study was approved by the Institutional Review Boards at the New York State Psychiatric Institute and Columbia University. Participants were paid for their participation.

Participants completed $\sim 3$ h of neuropsychological assessment with standardized psychometric measures evaluating intellectual functioning, attention, working memory, processing speed, executive functions, fine motor dexterity, and visual-motor integration in a controlled testing environment. Breaks were provided to minimize fatigue. Mothers completed questionnaires about demographics, education, home environment, and material hardship, and standardized surveys of their children's social, emotional, and behavioral functioning. The assessment was performed in a single session.

Neuropsychological tests were administered by research assistants trained on the administration and scoring of each measure by a pediatric neuropsychologist. From the extensive neuropsychological constructs assessed in the larger study, we selected raw scores of the following measures for correlation analyses, because they were most relevant to testing our a priori hypotheses of brain-based mediation of age-related improvements in cognitive performance in the peripubertal period: (1) The Conners' Continuous Performance Test, Second Edition (CPT-II; Conners, 1994). This is a 15 min computerized test of basic sustained attention, attentional capacity, and inhibitory control. The CPT-II provides individual performance scores for a range of cognitive processes including inattention (omission errors), inhibition and impulsivity (commission errors), reaction time, and the ability to discriminate targets from nontargets $\left(d^{\prime}\right)$. (2) The Developmental Neuropsychological Assessment, Second Edition (NEPSY-II; Kirk and Kemp, 2007). This is a standardized battery of tests of executive functions, language, learning, sensorimotor functions, visuospatial processing, and social perception. From this battery, we selected the Auditory Attention, Response Set, Inhibition, Inhibition/Switching, and Design Copying subtests. These measures assess selective and sustained auditory attention, response inhibition, cognitive flexibility, and visuospatial processing, respectively.

\section{Imaging measures}

DTI indices included FA and ADC. FA is a measure of directionally dependent diffusion, where higher values reflect increased structural integrity and degree of alignment within white matter fiber tracts (Cercignani et al., 2001; Mukherjee et al., 2002). ADC measures diffusion independent of direction. Radial diffusivity (RD) and axial diffusivity (AD) are two additional directional measures, with RD indicating diffusion rate perpendicular to the principal direction of diffusion, and $\mathrm{AD}$ indicating diffusion rate along the same direction as the principal (Feldman et al., 2010).

MPCSI quantifies brain metabolites to assess cellular function and metabolism (Goh et al., 2014). NAA is a putative index of the density of viable neurons, and choline $(\mathrm{Ch})$ is a marker of membrane turnover and may reflect alterations in cell density, creatine $(\mathrm{Cr})$ is a marker of metabolic activity (Rae, 2014), and glutamine (Glx) is an index of energy metabolism and neurotransmission (Ramadan et al., 2013).

\section{MRI data acquisition}

DTI and MPCSI data were acquired using a 3.0T Signa MR Scanner (GE Healthcare) equipped with an 8-channel receive head coil. Extra time was allowed on the morning of the scan to acclimate the participant to the MRI and its noise. Anxious children were calmed with frequent reminders, praise, and relaxation techniques, allowing for acquisition of motionfree data. During the scan, high-quality images were assured in real time with strict oversight of the data appearing on the console. If motion artifact was

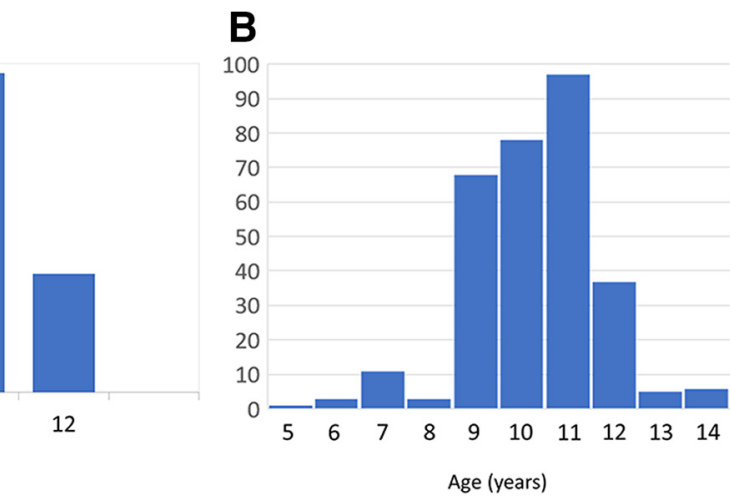

sequences included the following:

Anatomical MRI. High-resolution T1-weighted (T1w) anatomical images were acquired with a $3 \mathrm{D}$ fast-spoiled gradient recall sequence with sagittal reconstruction: repetition time $(\mathrm{TR})=2170 \mathrm{~ms}$, echo time $(\mathrm{TE})=1.3 \mathrm{~ms}$, inversion time $=500 \mathrm{~ms}$, flip angle $=11^{\circ}$, matrix $=$ $256 \times 256$, field-of-view $(\mathrm{FOV})=25 \mathrm{~cm}$, phase FOV $(\mathrm{PFOV})=100 \%$, slice thickness $=1.0 \mathrm{~mm}$, acceleration factor $=2$, number of slices $=160$, voxel size $=1 \times 1 \times 1 \mathrm{~mm}^{3}$. Two images each of NEX $=1$ were acquired and then averaged offline.

Diffusion tensor imaging. DTI data were acquired in oblique slices parallel to the AC-PC line using single-shot echoplanar imaging sequences with matrix $=132 \times 128$ zero-padded to $256 \times 256, \mathrm{TR}=8000 \mathrm{~ms}, \mathrm{TE}=$ $\sim 74 \mathrm{~ms}, \mathrm{FOV}=24 \mathrm{~cm}$, flip $=90^{\circ}$, slices $=60$, slice thickness $=2.5 \mathrm{~mm}$, slice spacing $=0 \mathrm{~mm}$; $\mathrm{NEX}=2, \mathrm{PFOV}=1.0$. Three baseline images were acquired with $b=0 \mathrm{~s} / \mathrm{mm}^{2}$ and 15 diffusion-weighted images (DWIs) with $b=1000 \mathrm{~s} / \mathrm{mm}^{2}$ along directions that sampled the space uniformly. Phase correction and ASSET acceleration factor of 2 were applied.

Multiplanar chemical shift imaging. Data were acquired in six axial oblique slices parallel to the AC-PC line, with the second bottom-most slice containing the AC-PC plane. Pulse sequence parameters included $\mathrm{TE}=144 \mathrm{~ms}, \mathrm{TR}=2800 \mathrm{~ms}, \mathrm{FOV}=24 \mathrm{~cm}$, slice thickness $=10.0 \mathrm{~mm}$, slice spacing $=2.0$, number of phase encoding steps $=24 \times 24, \mathrm{NEX}=$ 1 , spectral width $=2000 \mathrm{~Hz}$, number of complex data points $=512$. Water signal was suppressed using the CHESS sequence and lipid signal from outside the brain was suppressed with eight angulated saturation bands around the brain. MPCSI data were spatially registered to a template brain using a localizer image of high in-plane resolution in the same orientation and slice locations as the MPCSI data. Those images were acquired with $\mathrm{TR}=300 \mathrm{~ms}, \mathrm{TE}=10 \mathrm{~ms}, \mathrm{FOV}=24 \mathrm{~cm}$, slice thickness $=$ $10.0 \mathrm{~mm}$, spacing $=2.0 \mathrm{~mm}$, acquisition matrix $=256 \times 128$, image zero-padded to $256 \times 256$.

\section{Anatomical MRI segmentation}

The high-resolution T1-weighted anatomical images were segmented into gray and white matter for use in partial volume correction of MPCSI data. Brain was isolated from non-brain tissue using an automated brain extraction tool (BrainSuite; RRID:SCR_006623; Shattuck and Leahy, 2002) followed by manual editing using an in-house generated program to remove any dura that remained connected to the brain. Next, a thresholding technique based on a representative sampling of gray and white matter values was used to segment brain tissue as gray or white matter. These anatomical images were then registered across participants in template space. Segmentation and registration were completed using inhouse codes described previously (Colibazzi et al., 2008).

\section{DTI processing}

We conducted quality assurance procedures to exclude datasets with excessive motion. This included quantifying head motion for each participant using 2 summary statistics, the root mean squared (Jenkinson et al., 2002) and mean framewise displacement metrics, both of which sum 


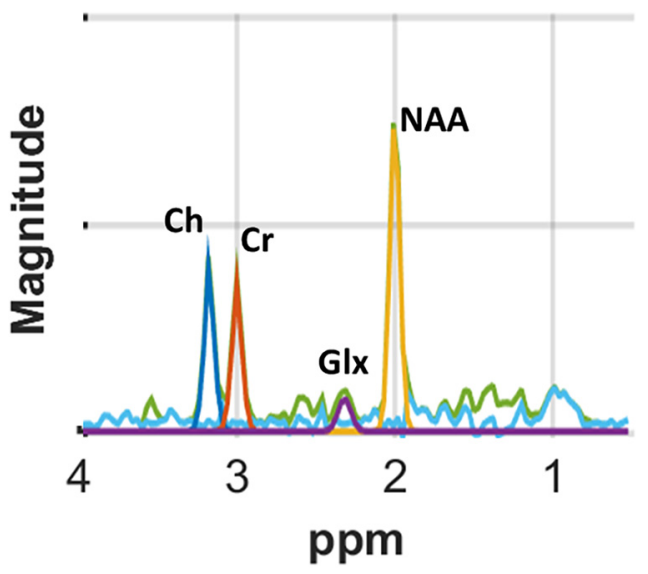

Figure 2. MR spectrum of sample voxel using a Lorentz-Gaussian line shape, principal resonances were fit for NAA plus N-acetyl-aspartyl-glutamate (2.0 ppm; yellow), Cr plus phosphocreatine (3.0 ppm; red), Ch compounds ( $3.2 \mathrm{ppm}$; dark blue), and glutamine + Glx (2.3 ppm; purple), as well as contaminating lipids (other colors). The quality of the spectra is excellent and signal-to-noise, especially for $\mathrm{NAA}, \mathrm{Cr}$, and $\mathrm{Ch}$, is high.

differentiated realignment estimates (Power et al., 2012) derived from three translational $(x, y, z)$ and three angular rotation (roll, pitch, yaw) variables. Images with $>0.5 \mathrm{~mm}$ motion were removed from further preprocessing; if $>10 \%$ of images had this much motion, that dataset was excluded from further processing. Next, we used quadratic warping along the anterior-posterior direction to correct for spatial distortions induced by eddy currents in the phase-encoding direction (Haselgrove and Moore, 1996). We also assessed motion by constructing tensor color maps from the retained images and visually assessing the principal eigenvectors throughout the brain, which show a color bias in the presence of motion artifact.

From the preprocessed data, we computed the measures for a priori hypothesis testing, FA and ADC, as well as secondary measures, $\mathrm{AD}$ and RD, voxelwise across the brain (DSI Studio; RRID:SCR_009557). A diffusion tensor $D$ was fit to the DWI data to ensure positive definiteness of the fitted tensor by first decomposing the tensor $D$ as the product $D=$ $\mathbf{A} \times \mathbf{A}^{\mathrm{T}}$ to estimate matrix $\mathbf{A}$, and then using the estimated matrix $\tilde{A}$ to compute the positive definite tensor $\tilde{D}=\tilde{A} * \tilde{A}^{T}$. The positive definite tensor was decomposed into its eigenvalues $\left(\lambda_{1}, \lambda_{2}, \lambda_{3}\right)$ and eigenvectors $\left(v_{1}, v_{2}, v_{3}\right)$, which were then used to compute the following:

$$
\begin{gathered}
F A=\frac{\sqrt{\left(\lambda_{1}-\lambda_{2}\right)^{2}+\left(\lambda_{2}-\lambda_{3}\right)^{2}+\left(\lambda_{3}-\lambda_{1}\right)^{2}}}{\sqrt{2 *\left(\lambda_{1}^{2}+\lambda_{2}^{2}+\lambda_{3}^{2}\right)}}, \\
A D C=\frac{1}{3}\left(\lambda_{1}+\lambda_{2}+\lambda_{3}\right), A D=\lambda_{1},
\end{gathered}
$$

and

$$
R D=\frac{1}{2}\left(\lambda_{2}+\lambda_{3}\right)
$$

FA, our primary measure, depicts the degree of directionality of water diffusion and is thought to represent the degree of local organization of white matter fibers and integrity of tissue microstructure (Alexander et al., 2007). ADC, also a primary measure, represents the magnitude of water diffusion, whereas $\mathrm{AD}$ and $\mathrm{RD}$ describe, respectively, the magnitude of diffusion parallel and perpendicular to the primary axis of the diffusion tensor. When considered together, maps of FA, ADC, RD, and $\mathrm{AD}$ aid interpretation of the biological basis for DTI findings.

Spatial normalization of DTI data. DTI data and its derived measures from all participants were spatially coregistered into the coordinate space of a template brain. This template was an artifact-free DTI dataset from a single individual of the same age as the modal age for all participants in the cohort. Each participant brain was coregistered to the template by applying an affine transformation ( 3 translations, 3 rotations, and 3 scaling parameters) to the participant brain that maximized the mutual information (Viola and Wells, 1995) between the B0 images of the participant and the template brains. Then the coregistered participant's B0 image was warped to the template B0 image using a high-dimensional, nonlinear deformation based on fluid flow dynamics (Christensen et al., 1994). Finally, the same affine transformation and nonlinear deformation were applied to register the participant's $\mathrm{FA}, \mathrm{ADC}, \mathrm{RD}$, and $\mathrm{AD}$ maps to the template.

\section{MPCSI processing}

MRS data quality was assured by reconstructing the data, assessing it for excess noise, and examining the spectrum in each voxel for baseline distortions, signal contamination by lipid signal from the scalp, incorrect placement of suppression bands, or broadening of line width. Sequences containing any of these artifacts were not processed further. We processed the signal from each coil of the 8-channel head coil separately before combining their processed MRS signals to generate the spectroscopic images (Dong and Peterson, 2007). First we phase-aligned signals, then smoothed the aligned signals using a Hamming window filter, spa-

\begin{tabular}{|c|c|c|}
\hline Characteristic & Age $9-12$ years $(n=280)$ & Age $5-14$ years $(n=309)$ \\
\hline Age, years & $10.86 \pm 0.99$ & $10.77 \pm 1.40$ \\
\hline \multirow[t]{2}{*}{ Gender } & Female, $148(52.9 \%)$ & Female, 165 (53.4\%) \\
\hline & Male, $132(47.1 \%)$ & Male, $144(46.6 \%)$ \\
\hline \multirow[t]{2}{*}{ Race/ethnicity } & Dominican/Dominican American, 168 (60\%) & Dominican/Dominican American, 186 (60.2\%) \\
\hline & African American, 112 (40\%) & African American, 123 (39.8\%) \\
\hline WISC FSIQ & $94.8 \pm 12.1$ (range $69-123)$ & $95.0 \pm 12.2$ (range $54-129)$ \\
\hline \multirow[t]{5}{*}{ Maternal Education, years } & $\leq 8$ th grade, $5(1.9 \%)$ & $\leq 8$ th grade, $5(1.6 \%)$ \\
\hline & 9 th to $<12$ th grade, $58(20.7 \%)$ & 9 th to $<12$ th grade, $64(20.7 \%)$ \\
\hline & High school graduate, 73 (26.1\%) & High school graduate, 82 (26.5\%) \\
\hline & $1-4$ years of college, $123(43.8 \%)$ & $1-4$ years of college, $137(44.3 \%)$ \\
\hline & $1-4$ years of graduate education, 21 (7.5\%) & $1-4$ years of graduate education, 21 (6.9\%) \\
\hline \multirow[t]{4}{*}{ Material Hardship Score ${ }^{a}$} & $0,167(59.6 \%)$ & $0,182(58.9 \%)$ \\
\hline & $1,59(21.1 \%)$ & $1,66(21.4 \%)$ \\
\hline & $2,54(19.3 \%)$ & $2,61(19.7 \%)$ \\
\hline & $3-4,0$ & $3-4,0$ \\
\hline
\end{tabular}
tially reconstructed the time-domain free induction decay (FID) signal in

Table 1. Demographics of study population

Demographics of study population, including entire cohort of 309 children ages 5-14 and 280 children ages $9-12$, with age of child at study, gender, race/ethnicity, WISC Full Scale IQ score, level of maternal education, and measure of maternal material hardship.

${ }^{a}$ Mothers were asked, "In the past year has there been a time when you: (1) couldn't afford to buy food?; (2) couldn't afford a place to stay?; (3) couldn't afford gas/electricity?; or (4) couldn't afford clothing?" Each positive response to one of the four questions was scored with 1 point. 
Table 2. Demographics of study population by imaging modality

\begin{tabular}{|c|c|c|c|c|}
\hline Characteristic & Age $9-12$ years & Range & Age $5-14$ years & Range \\
\hline \multicolumn{5}{|l|}{ Age, years } \\
\hline DTI only & $10.10 \pm 0.95(n=66)$ & $9.1-12.8$ & $10.14 \pm 1.48(n=73)$ & $5.9-14.1$ \\
\hline MRS only & $10.92 \pm 1.01(n=46)$ & $9.3-12.9$ & $10.79 \pm 1.17(n=48)$ & $7.3-12.9$ \\
\hline \multicolumn{5}{|l|}{ Gender } \\
\hline \multirow[t]{2}{*}{ DTI only } & Female, 35 (53\%) & & Female, 40 (54.8\%) & \\
\hline & Male, $31(47 \%)$ & & Male, $33(45.2 \%)$ & \\
\hline \multirow[t]{2}{*}{ DTI \& MRS } & Female, 93 (55.4\%) & & Female, 104 (55.3\%) & \\
\hline & Male, 75 (56.5\%) & & Male, 84 (44.7\%) & \\
\hline \multicolumn{5}{|l|}{ Race/ethnicity } \\
\hline \multirow[t]{2}{*}{ DTI only } & Dominican/Dominican American, 44 (66.7\%) & & Dominican/Dominican American, 50 (66.5\%) & \\
\hline & African American, 22 (33.3\%) & & African American, 23 (31.5\%) & \\
\hline MRS only & Dominican/Dominican American, 23 (50\%) & & Dominican/Dominican American, 23 (47.9\%) & \\
\hline DTI only & $91.5 \pm 11.3$ & $71-117$ & $91.7 \pm 11.2$ & $78-109$ \\
\hline MRS only & $93.6 \pm 13.2$ & $54-123$ & $93.1 \pm 13.4$ & $71-91$ \\
\hline DTI \& MRS & $96.4 \pm 11.8$ & $60-120$ & $96.8 \pm 12.0$ & $75-129$ \\
\hline \multicolumn{5}{|c|}{ Maternal education, years } \\
\hline \multirow[t]{5}{*}{ DTI only } & $\leq 8$ th grade, $1(1.5 \%)$ & & $\leq 8$ th grade, $1(1.4 \%)$ & \\
\hline & 9th to $<12$ th grade, $16(18.2 \%)$ & & 9th to $<12$ th grade, $17(23.3 \%)$ & \\
\hline & High school graduate, 11 (16.7\%) & & High school graduate, 14 (19.1\%) & \\
\hline & $1-4$ years of college, $30(45.5 \%)$ & & $1-4$ years of college, $33(45.2 \%)$ & \\
\hline & $1-4$ years of graduate education, 8 (12.1\%) & & $1-4$ years of graduate education, $8(11 \%)$ & \\
\hline \multirow[t]{3}{*}{ MRS only } & $\leq 8$ th grade, $0(0 \%)$ & & $\leq 8$ th grade, $0(0 \%)$ & \\
\hline & 9th to $<12$ th grade, $11(23.9 \%)$ & & 9th to < 12 th grade, $11(12.5 \%)$ & \\
\hline & High school graduate, 12 (26.1\%) & & High school graduate, 13 (27.1\%) & \\
\hline \multicolumn{5}{|c|}{ Material hardship Score $^{a}$} \\
\hline \multirow[t]{4}{*}{ DTI only } & $0,35(53 \%)$ & & $0,36(49.3 \%)$ & \\
\hline & $1,12(18.2 \%)$ & & $1,14(19.2 \%)$ & \\
\hline & $2,19(28.8 \%)$ & & $2,23(31.5 \%)$ & \\
\hline & $3-4,0$ & & $3-4,0$ & \\
\hline \multirow[t]{4}{*}{ MRS only } & $0,26(56.5 \%)$ & & $0,28(58.3 \%)$ & \\
\hline & $1,13(28.3 \%)$ & & $1,13(27.1 \%)$ & \\
\hline & $2,7(15.2 \%)$ & & $2,7(14.6 \%)$ & \\
\hline & $3-4,0$ & & $3-4,0$ & \\
\hline \multirow[t]{4}{*}{ DTI \& MRS } & $0,106(63.1 \%)$ & & $0,118(62.8 \%)$ & \\
\hline & $1,34(20.2 \%)$ & & $1,39(20.7 \%)$ & \\
\hline & $2,28(16.7 \%)$ & & $2,31(16.5 \%)$ & \\
\hline & $3-4,0$ & & $3-4,0$ & \\
\hline
\end{tabular}

Comparison of study population demographics for entire cohort (309) and narrower age range cohort (280), based upon type of imaging completed, DTI, MRS, or both DTI and MRS.

${ }^{a}$ Mothers were asked, "In the past year has there been a time when you: (1) couldn't afford to buy food?; (2) couldn't afford a place to stay?; (3)couldn't afford gas/electricity?; or (4) couldn't afford clothing?" Each positive response to one of the four questions was scored with 1 point.

each slice with a 2D Fourier transform, suppressed residual water signal by applying a high-pass filter to the FID signal, performed line broadening using a $4 \mathrm{~Hz}$ Gaussian filter, and then transferred the time-domain signal into the frequency domain with a 1D Fourier transform (Hao et al., 2013). Finally, the processed frequency-domain signal from each of the eight coils was combined by computing their weighted sum (MATLAB; RRID:SCR_001622). The combined signal was then loaded into the software 3DiCSI (3D Interactive Chemical Shift Imaging; RRID: SCR_002581) to identify MRS voxels within the brain and save spectral data for those voxels. Spectral fitting was applied to the frequencydomain signal by identifying peaks for NAA, Cr, Ch, glutamate + Glx, and lipids, with a modeling spectrum for those peaks of GaussianLorentzian curves. The areas under the fitted curves provided estimated concentrations of metabolites for each voxel of the brain.

Background noise for the MRS spectrum was computed as the SD of the real part of the complex spectrum free from the metabolite signal. We then calculated the signal-to-noise ratio (SNR) for each metabolite concentration. The average SNR for the NAA metabolite was $>280$; an excellent SNR, and attributable to use of the 8-channel head coil. A spectroscopic image for each metabolite was generated next as the ratio of the peak area to the background noise for each MRS voxel within the brain. We used the ratio of the peak area to the background noise as our 


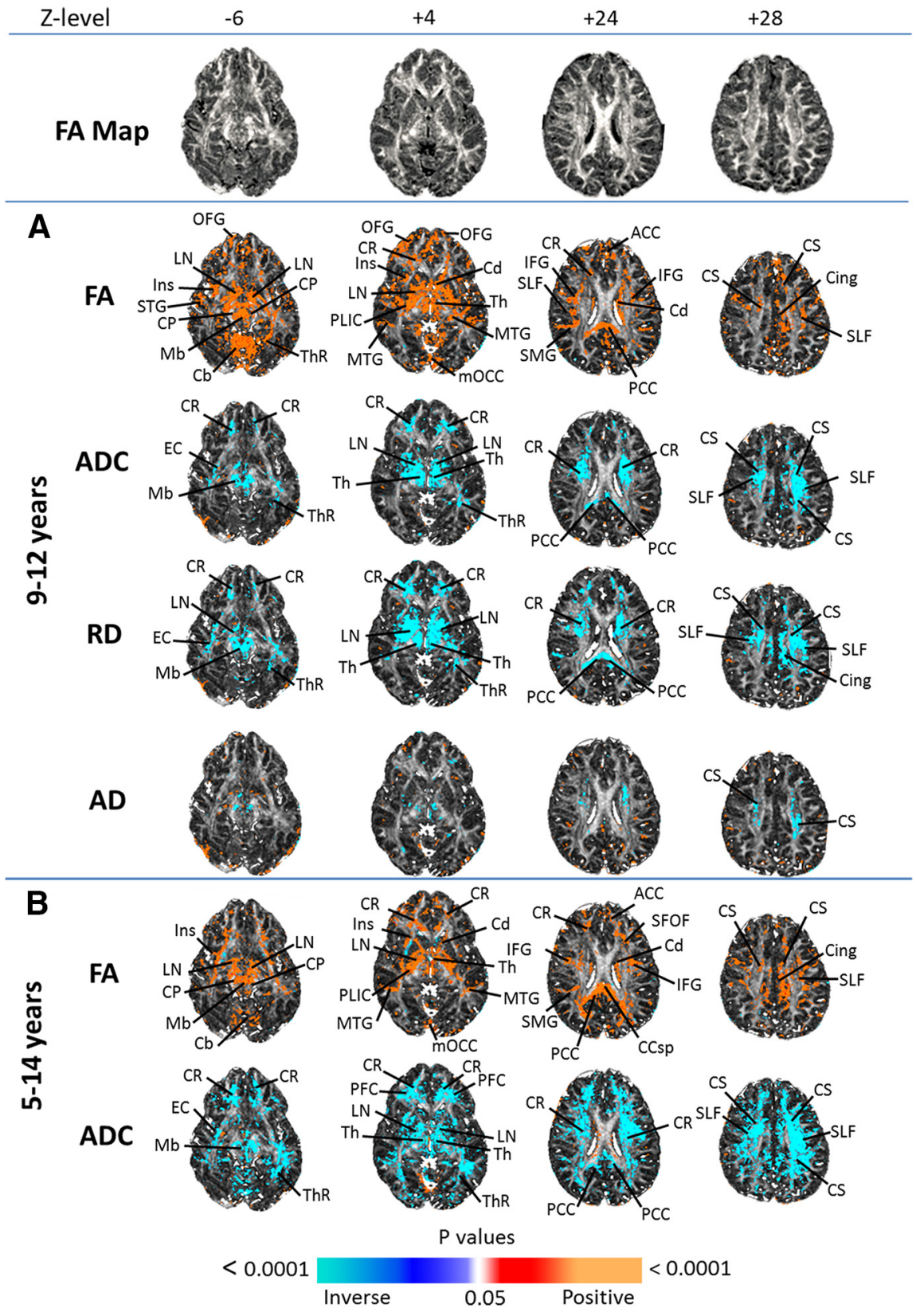

Figure 3. DTI age correlations axial brain DTI correlations with age, controlling for sex with FA average reference slices at top. $A$, Findings in the 9- to 12-year-old cohort, and $\boldsymbol{B}$ shows findings in the full 5- to 14-year-old cohort. FDR correction for multiple comparisons was applied with FDR at $p<0.01$. Significant positive correlations $(p<0.01)$ are indicated by warm colors (red, orange) and significant inverse correlations are shown as cool colors (shades of blue). FA correlated with age in the prefrontal cortex, deep WM, subcortical GM, and cerebellum. $A D C$ and $R D$ correlated inversely with age in bilateral thalami, prefrontal cortex, and temporal and frontal poles. Cb, Cerebellum; $\mathrm{Cd}$, caudate; cing, cingulate gyrus; $\mathrm{CP}$, cerebral peduncles; $\mathrm{CR}$, corona radiata; $\mathrm{CS}$, centrum semiovale; $\mathrm{EC}$, external/extreme capsule; IFG, inferior frontal gyrus; Ins, insular cortex; LN, lenticular nucleus; Mb, midbrain; mOCC, medial occipital cortex; MTG, middle temporal gyrus; OFG, orbitofrontal gyrus; PCC, posterior cingulate cortex; PLIC, posterior limb of internal capsule; SMG, supramarginal gyrus; STG, superior temporal gyrus; Th, thalamus; ThR, thalamic radiation.

dependent measure when assessing the age correlates of each metabolite concentration because it accounted for variations in receiver and transmitter gain within the MRS data across participants. Because we suppressed water signal, we were unable to calculate metabolite ratios using water as the reference. We elected to measure each neurometabolite individually, rather than as a ratio to $\mathrm{Cr}$ (a common practice in spectroscopy research), for several reasons. First, ratios multiply noise in the numerator and denominator and are generally undesirable for statistical analyses. Second, ratios may lead to erroneous inferences in developmental studies because both the numerator and denominator can vary with age, in which case interpreting the age correlates of the ratio requires that the effects of the numerator and denominator be assessed separately to understand which metabolite is driving the ratio findings. Our use of metabolite concentrations normalized to noise values overcomes these difficulties and provides a much clearer understanding of the age correlates of brain metabolite levels.

The spectroscopic images were also processed to correct for both partial volume effects within each MRS voxel and the spread of MRS signal of each voxel to its neighboring voxels [i.e., the point spread function (PSF)]. The spread of MRS signal from one voxel to its neighbors derives from the use of a small number of $k$-space samples when acquiring the spectroscopic data and from smoothing the data with a Hamming window before spatial reconstruction. We estimated the PSF by simulating the acquisition of MRS data within $k$-space on a $24 \times 24$ grid and then spatially filtering the simulated data using a Hamming window. The resulting $24 \times 24$ complex array was interpolated to $256 \times 256$ to match the spatial resolution of the T1-weighted MR images. Partial volume effects reference the fact that an MRS voxel may contain more than one tissue type, i.e., gray matter (GM) and white matter (WM), in varying proportions; therefore, the signal in that voxel is a proportionate combination of signals from each of those tissues. To estimate the proportions of GM and WM within an MRS voxel, brain tissue was segmented as either GM or WM, as described, within the participant's high-resolution $\left(1 \mathrm{~mm}^{3}\right.$ voxel) T1-weighted image, and then coregistered to the MRS data of that participant (detailed in the next section). We then convolved the coregistered tissue definitions with the PSF function and computed the fractions of GM and WM within each MRS voxel. We next used a linear regression model (Lebon et al., 2002) at each voxel $i$ and for each metabolite $j$, along with the concentrations $S_{i j}$ of that metabolite in the neighboring voxels, to estimate the concentration of that metabolite within gray matter $M_{i j}^{G}$ and white matter $M_{i j}^{W}$ :

$$
S_{i j}=\left|c_{i}^{G} * M_{i j}^{G}+c_{i}^{W} * M_{i j}^{W}\right|+n,
$$

where $c_{i}^{G}$ and $c_{i}^{W}$ are the proportions of GM and WM, respectively, at voxel $i$ and $n$ is noise. We then tri-linearly resampled the metabolite concentrations $M_{i j}^{G}$ and $M_{i j}^{W}$ from lowresolution MRS data to high-resolution anatomical data for spatial normalization across study participants. Figure 2 shows a representative spectrum in a voxel of the MPCSI dataset.

Spatial Normalization of MPCSI Data. MPCSI data for each participant were coregistered into the coordinate space of a T1-weighted image of a template brain. Specifically, each participant's localizer image was coregistered to its high-resolution T1w image using a similarity transformation ( 3 translations and 3 rotations) such that the transformation maximized mutual information (Viola and Wells, 1995) across the localizer and its corresponding high-resolution T1w image. Second, we spatially transformed the localizer image using the similarity transformation that coregistered the T1w image of the participant into the coordinate 
space of the template brain. Third, we warped the coregistered localizer by applying to it the high-dimension, nonlinear deformation that warped the participant $\mathrm{T} 1 \mathrm{w}$ image to the template T1w image. We applied these three coregistration procedures to each of the metabolite images.

The MPCSI saturation bands applied to suppress lipid signal from the scalp were not as precisely shaped as the scalp, and they unavoidably suppressed metabolite signals from several portions of cortical gray matter. Moreover, lipid signal from the small portions of scalp that were unsuppressed contaminated MRS signal to some degree within the brain, and those voxels were censored from further analyses during the detailed visual inspection of the spectra recorded from each of the eight channels of the multichannel head coil. Consequently, metabolite measures for many participants were available only in voxels of WM and deep GM nuclei. We show in each metabolite map a gray scale image representing the number of participants who had usable data at each voxel. We suppressed display of results at any voxel that did not have usable data from at least one-half the participants.

\section{Statistical analyses}

Age correlates of DTI and MPCSI measures. We hypothesized that increasing age would be associated with differences in cerebral microstructure (FA, ADC) and neurometabolite concentrations (NAA) in CSTC pathways; representing progressively advancing brain maturation within our primary cohort of children ages 9-12. To test these hypotheses, we used voxelwise multiple linear regression models in MATLAB (MathWorks; RRID:SCR_001622) using in-house generated programs to assess the association of age with DTI (FA and ADC) and MPCSI (NAA) measures entered separately as dependent variables, age entered as the independent variable, and sex as a covariate.

Mediation analyses. We assessed whether our imaging measures mediated the association of age with measures of psychometric performance. These analyses would only be meaningful when applied to psychometric variables that correlated significantly with age. Therefore, we assessed mediation only for those performance raw scores (i.e., unadjusted for age) that correlated significantly with age while controlling for sex. Raw scores that were highly skewed were transformed using an inverse hyperbolic sine: $\operatorname{ihs}(x)=\log \left[x+\sqrt{x^{2}+1}\right]$ (Burbridge et al., 1988). Of the raw performance scores that correlated significantly with age, only NEPSY Inhibition/Switching and CPT Hit Reaction Time scores sufficiently normalized to provide stable mediation estimates and entered mediation analyses along with Design Copy scores, which did not require transformation. We then calculated the statistical significance of the brain imaging values (variable $M$ ) mediating the association between age (independent variable $X$ ), and psychometric scores (dependent variable $Y$ ), using three regression equations: (1) $Y=c_{1} X+e_{1}$, which examines the unadjusted association of age with psychometric score; (2) $M$ $=a X+e_{2}$ which examines the association of age with the brain mediator; and (3) $Y=c_{2} X+b M+e_{3}$, which examines the association of

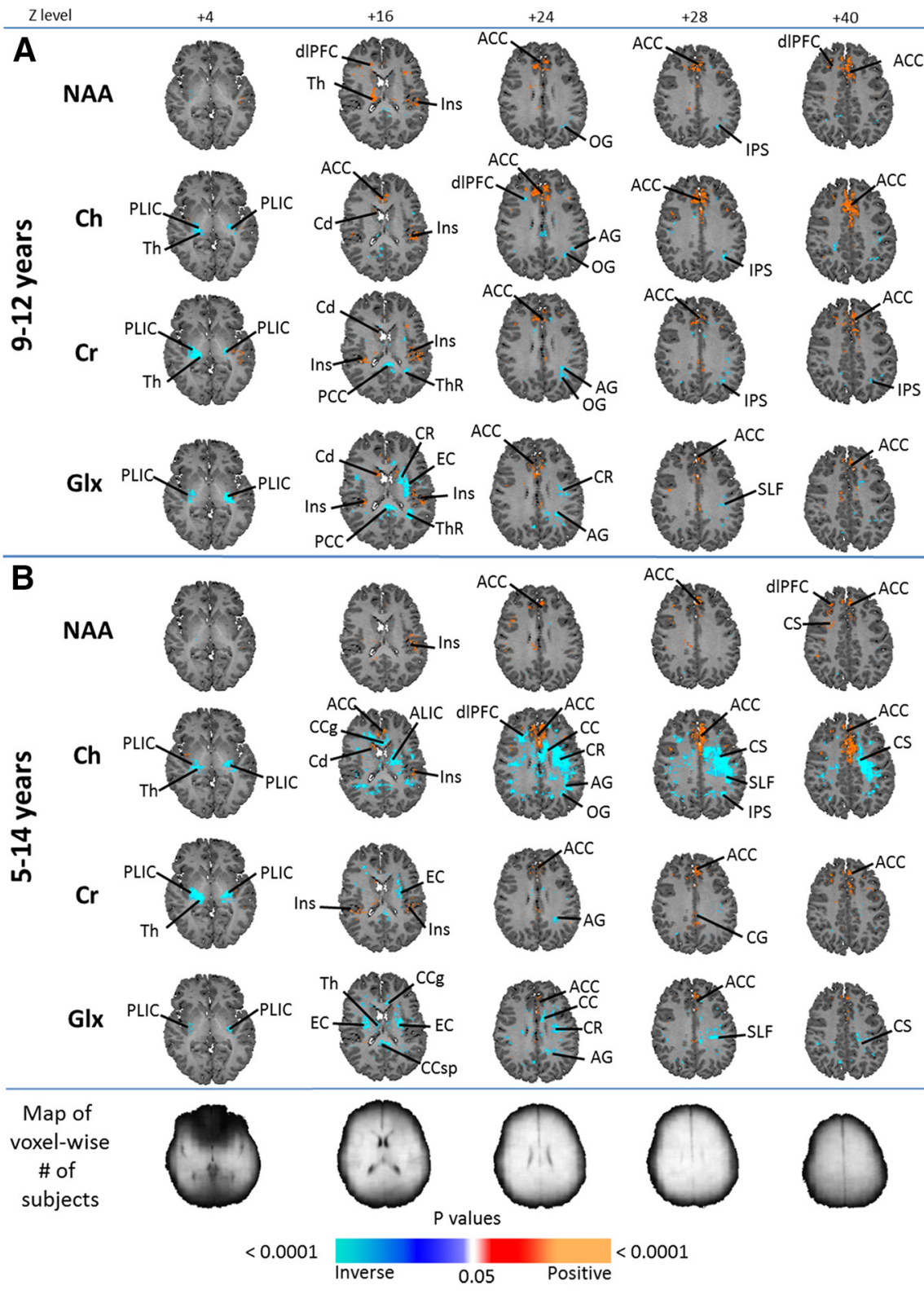

Figure 4. MPCSI age correlations shown are MPCSI metabolite correlations with age, controlling for sex, with anatomical reference slices at the top and maps indicating voxelwise numbers of subjects at the bottom, with lighter shading indicating more subjects. $A$, Results from the 9 - to 12 -year-old cohort, whereas $B$ shows results of the full 5 - to 14 -year-old cohort. FDR correction for multiple comparisons was done with FDR at $p<0.01$. Significant positive correlations $(p<0.01)$ are indicated by warm colors (red, orange) whereas significant inverse correlations are shown as cool colors (shades of blue). All metabolites, NAA, $\mathrm{Cr}$, Ch, and Glx correlated with age in the ACC in both age groups. NAA additionally correlated with age in the dIPFC, insular cortex, and thalamus; and inversely correlated with age in the intraparietal sulcus and occipital gyrus. Ch correlated with age in the insular cortex and caudate, and inversely correlated with age in the occipital and angular gyri, thalamus, IPS, and PLIC. Cr correlated with age in the caudate and insular cortex, and inversely correlated with age in the occipital and angular gyri, PCC, TR, thalamus, IPS and PLIC. Glx correlated with age in the insular cortex and caudate, and inversely with age in the posterior limb of the internal capsule, external capsule, corona radiata, angular gyrus, SLF, posterior cingulate cortex, and thalamic radiation. AG, Angular gyrus; CC, corpus callosum (body); $C(\mathrm{Cg}$, genu of corpus callosum; $\mathrm{Cd}$, caudate; $\mathrm{CR}$, corona radiata; $\mathrm{CS}$, centrum semiovale; EC, external capsule; Ins, insular cortex; IPS, intraparietal sulcus; OG, occipital gyrus; PCC, posterior cingulate cortex; PLIC, posterior limb of internal capsule; Th, thalamus; ThR, thalamic radiation.

age with psychometric score adjusting for the mediator. Sex was included as a covariate in all three equations. As noted above, we first assessed whether the independent variable (age) was significantly associated with the dependent variable (raw performance scores); we also assessed whether the independent variable (age) was significantly associated with the mediating variable (imaging measures). If both of these associations were significant, we then tested whether the indirect effect $a \times b$ differed significantly from zero using a $z$-score, 
$Z_{a b}=(a \times b) / s e_{a b}$, where $s e_{a b}=\sqrt{\left(a^{2} \times s e_{b}^{2}\right) \times\left(b^{2} \times s e_{a}^{2}\right)}$, and $s e_{a}, s e_{b}$, were the SEs of the regression coefficients $a$ and $b$, respectively (MacKinnon, 2008). Significant mediating effect suggests that association of $X$ with $Y$ in regression (1) is weakened by $M$ in regression (3).

Post hoc analyses. We conducted several post hoc tests to aid interpretation of findings from a priori hypothesis testing. For example, we performed linear regression using $\mathrm{AD}$ and $\mathrm{RD}$ as the dependent variables to interpret better the microstructural features likely generating the age correlations for FA and ADC. Similarly, linear regressions using $\mathrm{Ch}, \mathrm{Cr}$, and Glx as dependent variables aided interpretation of age correlates for NAA in terms of membrane turnover (Ch), energy metabolism $(\mathrm{Cr})$, or neurotransmission (Glx). Additional post hoc analyses included age correlations and mediation analyses for the entire cohort of 309 participants, aged 5-14 years, to assess whether findings in the restricted age range of 9-12 years obtained across a wider developmental window. We also performed regression analyses with family income, maternal hardship, and ethnicity entered as additional covariates to ensure that our findings were not attributable to possible confounds in this sample of participants. Finally, we generated scatterplots of significant correlations of age with brain imaging and psychometric measures to provide further assurance that findings were not spurious or generated by statistical outliers, and to assess whether age correlates contained any curvilinear effects.

Statistical parametric maps and correction for multiple comparisons. We used a method for false discovery rate (FDR) to control for multiple comparisons across all the voxels within each statistical map. We further corrected $p$ values for the five statistical tests of our a priori hypotheses, to establish an overall $p$ value $<0.05$ study-wide. Thus, we required the five maps that tested our five a priori hypotheses to yield an $\operatorname{FDR} p<0.01$. Furthermore, we eliminated regions with $<100$ significant voxels, assuming them to be less reproducible and biologically less important. We applied morphological operators of dilation followed by erosion, each two voxels wide, to fill two voxel-wide holes in thresholded $p$ value maps. $P$ values $<0.01$ that survived FDR correction were color-coded and plotted on brain images such that warm colors (red/orange) represented positive associations and cool colors (blue shades) indicated inverse associations.

We note that application of the FDR procedure across MRS and DTI data, resampled to the 557,200 voxels of the high-resolution anatomical image, in itself provides a stringent and conservative control for falsepositives in our findings because the measures were highly correlated across voxels. We acquired the MRS data, for example, on a $24 \times 24$ grid in each of the six axial-oblique slices parallel to the AC-PC line. The nominal, within-slice resolution was $10 \times 10 \mathrm{~mm}^{2}$, with $10 \mathrm{~mm}$ slice thickness. The signal from an MRS voxel within a slice, however, was dispersed into its neighboring voxels with a PSF having a full-width at half-maximum of $30 \mathrm{~mm}$, yielding a total of $6 \times(240 \times 240) /(30 \times$ 30) $=384$ independent resolution elements. Therefore, although we needed to control for $<400$ independent statistical tests for the MRS data, we applied the FDR procedure to 557,200 voxels to provide added confidence in the validity of our findings.

\section{Results}

Demographic data for both the 9 to 12 - and 5 to 14 -year-old age groups are presented in Table 1. Participants were exclusively racial/ethnic minorities and of lower to middle socioeconomic status.

A comparison of demographic data by imaging modality (participants with usable DTI, MRS, or both DTI and MRS) for both age cohorts is shown in Table 2.

\section{Age correlations with DTI measures \\ Fractional anisotropy}

White matter. FA correlated positively with age in deep white matter fiber bundles such as the corona radiata, posterior limb of the internal capsule, superior longitudinal fasciculus, posterior thalamic radiation (ThR), and cerebral peduncles, as well as in superficial cortical white matter within prefrontal and parietal regions (Fig. 3A). The ThR and superior longitudinal fasciculus (SLF) are deep WM fiber tracts that integrate informa-
Table 3. Significant correlations of neurocognitive scores ${ }^{a}$ with age

\begin{tabular}{|c|c|c|c|c|}
\hline Instrument & Task & Subscale & Correlation & $p$ \\
\hline \multirow[t]{18}{*}{ NEPSY } & \multirow[t]{2}{*}{ INI inhibition } & Total self-corrected errors ${ }^{b}$ & -0.16 & 0.02 \\
\hline & & Total uncorrected errors $^{b}$ & -0.19 & 0.01 \\
\hline & \multirow[t]{2}{*}{ INS switching } & Total errors ${ }^{b}$ & -0.18 & 0.01 \\
\hline & & Total commission errors ${ }^{b}$ & -0.23 & 0.001 \\
\hline & \multirow[t]{3}{*}{ Auditory Attention } & Total omission errors ${ }^{b}$ & -0.21 & 0.002 \\
\hline & & Total commission errors $^{b}$ & -0.30 & 0.0003 \\
\hline & & Total omission errors ${ }^{b}$ & -0.19 & 0.006 \\
\hline & \multirow[t]{2}{*}{ Response set } & Total inhibitory errors ${ }^{b}$ & -0.26 & 0.001 \\
\hline & & Uncorrected errors ${ }^{b}$ & -0.19 & 0.004 \\
\hline & \multirow[t]{5}{*}{ Naming } & Total errors & -0.15 & 0.03 \\
\hline & & Process total & 0.29 & $<0.0001$ \\
\hline & & Process local & 0.28 & 0.00002 \\
\hline & & Process global & 0.23 & 0.0005 \\
\hline & & Process motor & 0.24 & 0.0002 \\
\hline & \multirow[t]{4}{*}{ Design copy } & Global total & 0.21 & 0.002 \\
\hline & & 0mission errors & -0.13 & 0.05 \\
\hline & & Commission errors $^{b}$ & -0.17 & 0.01 \\
\hline & & Hit reaction time $e^{b}$ & -0.24 & 0.0004 \\
\hline Conners' CPT & Total overall & Detectability $d^{\prime}$ & 0.18 & 0.01 \\
\hline
\end{tabular}

Significant correlations of neurocognitive scores with age to demonstrate those measures that were included in the mediation analyses, and the direction of association of age with each score.

${ }^{a}$ All were raw scores, unadjusted for participant age or sex.

${ }^{b}$ When transformed, those raw scores also correlated with age.

tion across distant and disparate brain regions; anterior to posterior brain regions for the SLF, and from cortex to thalamus and brainstem for the TR.

Gray matter. FA correlated positively with age in the anterior and posterior cingulate cortices, superficial cortical gray matter, lenticular nucleus, caudate, thalamus, midbrain, medial occipital cortex, and cerebellum (Fig. 3A).

\section{Apparent diffusion coefficient}

White matter. ADC correlated inversely with age in the corona radiata, centrum semiovale, SLF, thalamic radiation, and extreme capsule, as well as in superficial cortical white matter in the PFC (Fig. $3 A$ ).

Gray matter. ADC correlated inversely with age in subcortical gray matter including the lenticular nucleus and thalamus, and in the posterior cingulate cortex and midbrain (Fig. 3A).

\section{Post hoc analyses}

$R D$ and $A D$. The similarity in RD maps to ADC maps, and the minimal correlations of age with $\mathrm{AD}$ for both $\mathrm{WM}$ and $\mathrm{GM}$, indicate that agerelated changes in the radial direction of diffusion accounted for correlations of age with ADC (Fig. 3A).

Extended age range. DTI findings in GM were consistent across the 9 to 12 - and 5 to 14-year-old cohorts, though they appeared somewhat stronger in WM in the 5-14 year olds (Fig. 3B).

\section{Age correlations with metabolite concentrations}

NAA

White matter. NAA correlated with age in the dlPFC and inversely with age in the parietal WM (Fig. 4A).

Gray matter. NAA correlated positively with age in the dorsal anterior cingulate cortex (ACC), insula, and thalamus, and inversely in the medial occipital GM (Fig. 4A).

\section{Post hoc analyses}

Ch

White matter. Ch correlated inversely with age in the PLIC, dlPFC, and parietal and occipital WM (Fig. 4A).

Gray matter. Ch correlated positively with age in the pregenual and dorsal ACC, caudate, and insular cortex (Fig. 4A). 
$\mathrm{Cr}$

White matter. Cr correlated inversely with age in the posterior limb of the internal capsule, thalamic radiation, and parietal WM (Fig. 4A).

Gray matter. Cr correlated positively with age in the dorsal ACC and insular cortex, and inversely in the thalamus (Fig. $4 A$ ).

Glx

White matter. Glx correlated inversely with age in the posterior limb of the internal capsule, external capsule, angular gyrus, corona radiata, SLF, and thalamic radiation (Fig. 4A).

Gray matter. Glx correlated positively with age in the insular cortex, caudate, and ACC (Fig. 4A).

Extended age range. The MRS findings in both WM and GM were similar in the 5- to 14-year-olds, except for stronger inverse correlations of age with $\mathrm{Ch}$ concentrations in deep WM (Fig. 4B).

\section{Brain measures mediating the association of age with cognitive performance}

We assessed whether brain imaging measures mediated the association of age with measures of performance on self-regulatory tasks, testing mediation only for those cognitive variables that correlated significantly with age (Table 3). Notably, all raw error scores and CPT Hit Reaction Time correlated significantly and inversely with age, thus indicating that increasing age was associated with decreasing errors and shorter reaction times, representing improved performance. CPT Hit Reaction Time Detectability and NEPSY Design Copy scores correlated positively with age, signifying improved performance with age.

\section{Fractional anisotropy}

FA significantly mediated the associations of age with the NEPSY Design Copy Process Global scores and CPT Hit Reaction Time. The number of participants varied slightly among different measures, as noted in Figures 5 and 6 , because not every participant with imaging completed each psychometric measure. Increased FA in GM of the thalamus and caudate, and WM of the internal capsule and splenium of the corpus callosum partially mediated the association of age with better scores on the Process Global subscale of the Design Copy task, which requires both visuospatial processing skills and executive functioning (Fig. 5A). Higher FA in the lenticular nucleus and thalamus, but also in the inferior fronto-occipital fasciculus (IFOF), internal capsule, and splenium of the corpus callosum partially mediated the association of increasing age with better performance (demonstrated as lower scores, or shorter Hit Reaction Times) on the CPT (Fig. 5A).

Post hoc analyses. In the 5- to 14-year-olds, mediation of FA with age in CPT Hit Reaction Time was significant in all areas but the IFOF, and in Design Copy was significant in the CCsp and caudate (Fig. 5B).
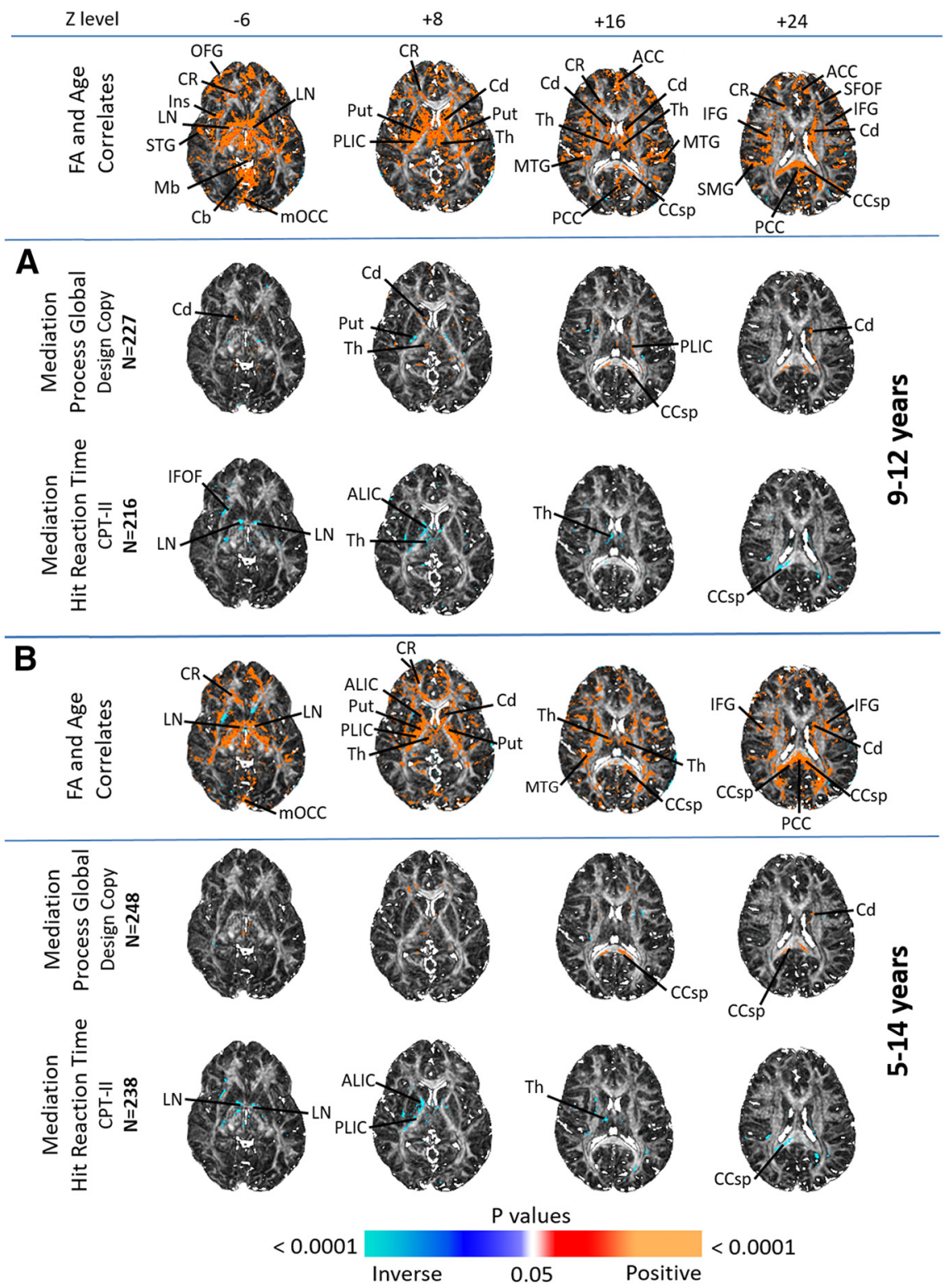

Figure 5. FA mediation shown are axial brain images of FA mediating the associations of age with psychometric scores; FA correlations with age are shown at the top for ease of reference. $\boldsymbol{A}$, Findings in the 9 - to 12 -year-old cohort; $\boldsymbol{B}$, findings in the 5 - to 14-year-old cohort. FDR correction for multiple comparisons was applied with FDR at $p<0.01$. Significant positive correlations $(p<0.01)$ are indicated by warm colors (red, orange), and significant inverse correlations are shown as cool colors (shades of blue). Significant FA mediations of age were seen with Design Copy Process Global and Hit Reaction Time scores, most notably in the lenticular nucleus, thalamus, caudate, posterior limb of the internal capsule, corpus callosum and inferior fronto-occipital fasciculus. ALIC, Anterior limb of internal capsule; Cb, cerebellum; CCsp, splenium of corpus callosum; Cd, caudate; CR, corona radiata; IFG, inferior frontal gyrus; Ins, insular cortex; LN, lenticular nucleus; Mb, midbrain; mOCC, medial occipital cortex; MTG, middle temporal gyrus; OFG, orbitofrontal gyrus; PCC, posterior cingulate cortex; PLIC, posterior limb of internal capsule; Put, putamen; SFOF, superior fronto-occipital fasciculus; SMG, supramarginal gyrus; STG, superior temporal gyrus; Th, thalamus.

NAA

NAA in the thalamus partially mediated the association of age with improving performance (shorter Hit Reaction Times) on the CPT (Fig. 6A).

Post hoc analyses. The NAA mediation finding in the thalamus was not detected in the 5- to 14-year-olds (Fig. 6B).

\section{Glx}

Glx in the WM of the putamen and external capsule partially mediated the association of age with improved performance on the CPT (Fig. 6C), and mediation in the putamen was also significant in the full cohort (Fig. 6D). 


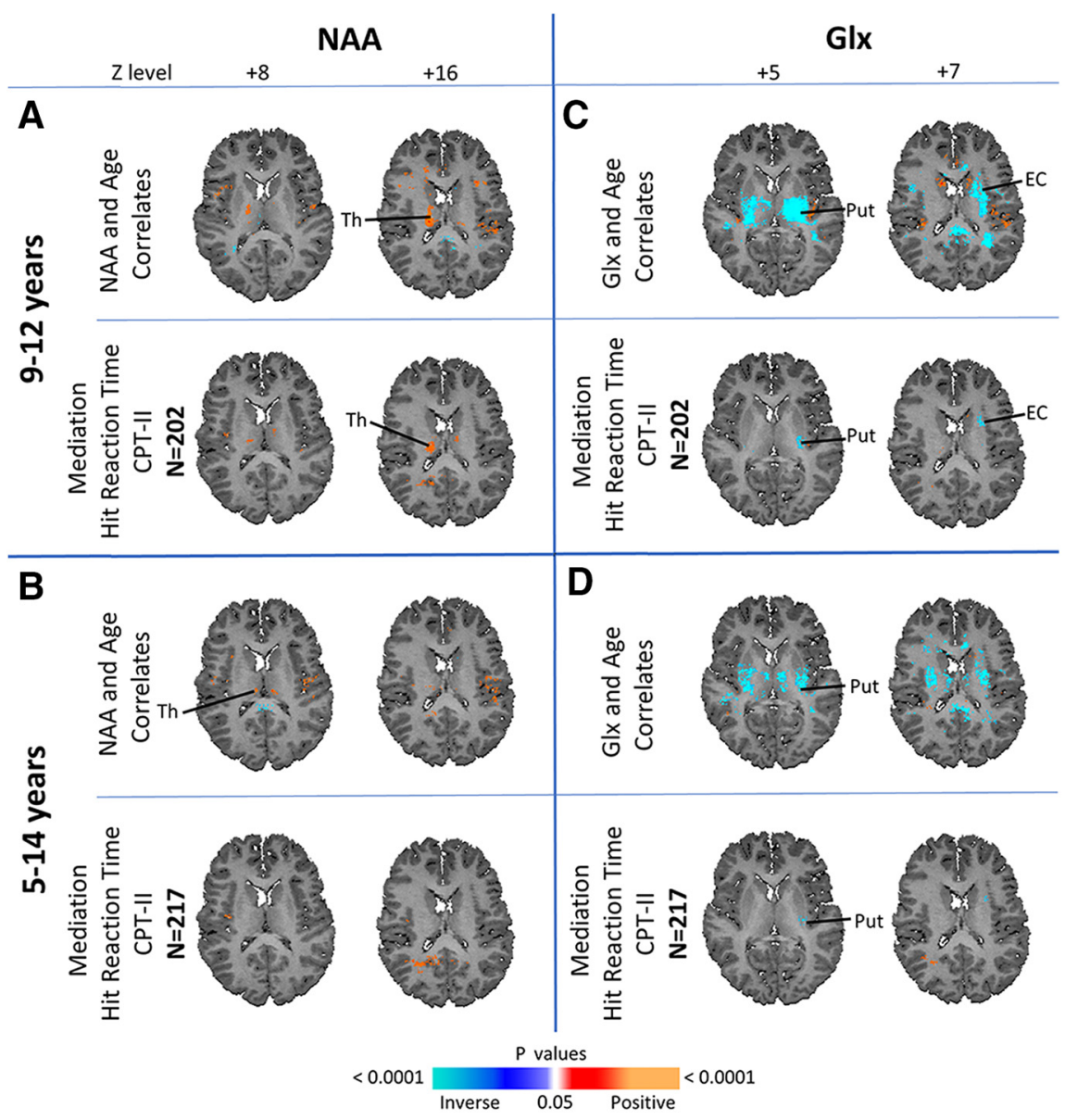

Figure 6. NAA and Glx mediation shown are axial brain images of NAA and Glx mediating associations of age with psychometric scores, NAA/Glx correlations with age are shown in $\boldsymbol{A}$ and $\boldsymbol{C}$ for ages $9-12$, and in $\boldsymbol{B}$ and $\boldsymbol{D}$ for ages $5-14$, for reference. Significant mediation by NAA in the thalamus was found for the association age with Hit Reaction Time scores in the $9-12$ year age group, but not in the expanded 5 - to 14-year-old sample. Significant mediation by Glx in the putamen and external capsule was found for the associations of age with Hit Reaction Time scores in the 9-12 age group, and in the putamen in the expanded 5-14-year-old sample. EC, External capsule; Put, putamen; Th, thalamus.

Diagrams of each mediation with scatterplots of representative brain regions are shown for FA (Fig. 7), and for NAA and Glx (Fig. 8), demonstrating age correlates that vary monotonically and positively with increasing age, without evidence of curvilinear effects. FA mediation of the association of age with CPT Hit Reaction Time in the restricted age group was significant in the PLIC at $p=0.00001$, unstandardized $\beta=$ -0.0003 (95\% confidence interval: $-0.0004,-0.0002$, effect size $=$ -2.99 ; Fig. $7 A$, top). In the full cohort, mediation was significant at $p=0.0002$, unstandardized $\beta=-0.0002$ [95\% confidence interval: $-0.0003,-0.0001$, effect size -2.65 (Cohen's $d$ effect size computed by dividing the mean of the mediation effect by its SD); Fig. 7B, top]. FA mediation of age correlates with Design Copy in the restricted age group was significant in the caudate at $p=0.01$, unstandardized $\beta=0.005$ ( $95 \%$ confidence interval: $0.001,0.01$, effect size $=1.61$; Fig. $7 A$, bottom); and was also significant in the expanded age group at $p=0.01$, unstandardized $\beta=0.004$ ( $95 \%$ confidence interval: $0.001,0.008$, effect size $=2.11$; Fig. $7 B$, bottom).

NAA mediation of the age correlate with CPT Hit Reaction Time was significant in the thalamus in the limited age cohort at $p=0.02$, unstandardized $\beta=0.0002$ (95\% confidence interval $0.00003,0.0003$, effect size $=1.82$; Fig. $8 A$, top), but there was no brain region finding of NAA mediation in the expanded cohort (Fig. 8B, top). Glx mediation of age correlates with CPT Hit
Reaction Time was significant in the putamen in both the $9-12$ year cohort $[p=$ 0.05 , unstandardized $\beta=0.0004$ (95\% confidence interval: $0.00001,0.0007$, effect size $=-1.48$ ); Fig. $8 A$, bottom] and full cohort $[p=0.05$, unstandardized $\beta=$ 0.0003 (95\% confidence interval: 0.000004 , 0.0007, effect size $=-1.55$ ); Fig. $8 B$, bottom].

\section{Discussion}

The transition from childhood to adolescence is characterized by an increasing ability to self-regulate thought, emotion, and behavior. Using voxelwise analyses of DTI and MPCSI data, we have shown that this period is also characterized by maturational correlates in microstructure and metabolites, most prominently in frontal and cingulate cortices, striatum, thalamus, WM, and cerebellum. Our results also show that maturational correlates in the striatum, thalamus, and WM partially mediate performance improvements in preadolescent children. These findings generally held true in post hoc analyses of an expanded cohort 5-14 years of age.

\section{White matter maturation}

Increasing age was associated with increasing FA and decreases in ADC and RD across large sectors of frontal WM, extending from the centrum semiovale to deep WM, and anteriorly to the frontal pole (Fig. 3). We found little evidence for age-related decreases in $\mathrm{ADC}, \mathrm{RD}$, or $\mathrm{AD}$ in parietal or occipital WM.

Strong positive associations of age with higher FA values, and inverse associations of age with $A D C$ and $R D$ values, with little contribution from $\mathrm{AD}$, suggest that cellular maturation disproportionately reduces diffusion in the radial direction of fiber bundles. The most obvious possible explanations for these findings include age-related increases in myelination or axon packing density. Myelination restricts radial diffusion in axons, primarily because myelin is highly hydrophobic (Stoffel et al., 1984); increasing myelination with age would reduce $\mathrm{ADC}$ and $\mathrm{RD}$, and increase FA. Greater axon packing density would yield more plasma membranes, myelin, and cell organelles, and less extracellular water, reducing RD and possibly increasing FA (Beaulieu, 2002).

Prior studies in children and adolescents reported age-related decreases in ADC and RD (Lebel et al., 2008; Colby et al., 2011; Simmonds et al., 2014) and age-related increases in FA (BarneaGoraly et al., 2005) in WM. These maturational changes, thought to reflect ongoing myelination (Yakovlev and Lecours, 1967; Colby et al., 2011), appear to follow a posterior-to-anterior temporal gradient. Our findings are consistent with these reports across a much narrower age-range (9-12 years), and they demonstrate, for the first time, that associations of age with microstructure are not accompanied by age-related alterations in WM metabolite concentrations (Fig. 4).

This observation differs markedly from the close association of myelination with metabolite concentrations observed 

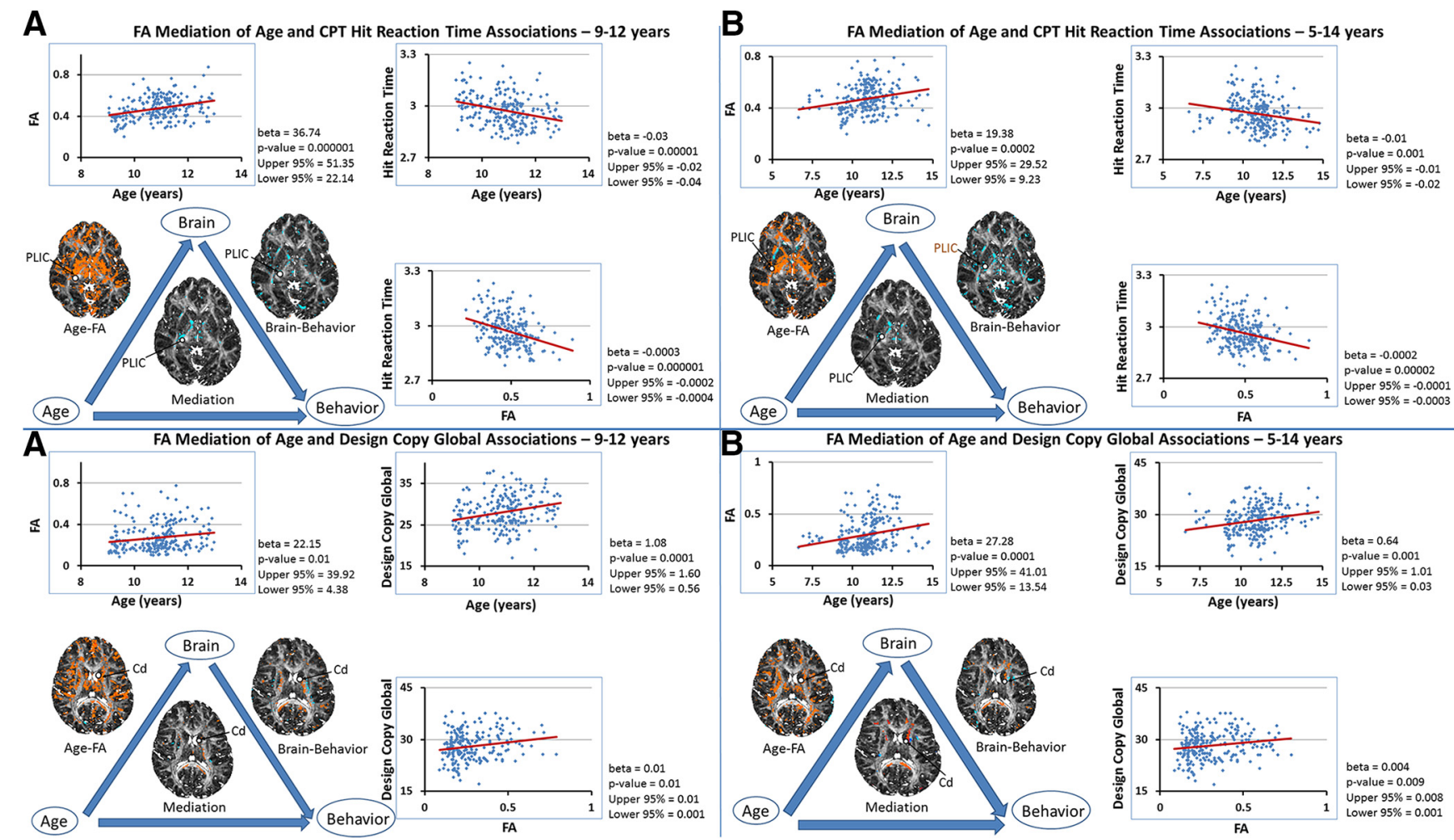

Figure 7. FA mediation with scatterplots shown are illustrations of the mediating relationship of FA with age and behavioral measures. Representative scatterplots of sampled brain regions are shown for $F A$ in children $(\boldsymbol{A})$ 9-12 years old and $(\boldsymbol{B})$ 5-14 years, where beta is the degree of change in FA per unit change in age. All associations were statistically significant. In each panel, the scatterplot on the top left shows the correlation of FA with age; that on the top right shows the correlation of behavioral measure (CPT Hit Reaction Time or Design Copy Process score) with age; and that on the bottom right shows the correlation of behavioral measure with FA. Effect size for $\boldsymbol{A}$ (top) was -2.99 and for $\boldsymbol{B}$ (top) was -2.65 . Effect size for $\boldsymbol{A}$ (bottom) was 1.61 and for $\boldsymbol{B}$ (bottom) was 2.11. Cd, Caudate; PLIC, posterior limb of internal capsule.

earlier in development (Blüml et al., 2013). Although unexpected, the absence of significant age correlates in WM metabolite concentrations, together with significant age correlations in WM microstructure, has at least two possible explanations. First, transient variation of metabolite concentrations during this developmental period could be missed by our linear models and cross-sectional study design. Second, WM microstructure development during this period may not require substantial metabolite alterations.

Myelin forms when an oligodendrocyte wraps an axon with a flattened cytoplasmic process. Over time, that process loses its cytoplasmic content to form a much more compact, tightly wound, membranous sheath of alternating lipid and protein laminae (Raine, 1984). After myelin synthesis peaks, protein and lipid synthesis in myelin decline rapidly (Benjamins and Smith, 1984), though slow turnover of the constituent myelin lipids and proteins, and activity-dependent remodeling of myelin, continues across the lifespan (Smith, 1968; Uzman and Hedley-Whyte, 1968; Nave, 2010; Young et al., 2013; Gibson et al., 2014; Yeung et al., 2014). Accordingly, our findings of an age-related decline in RD and increase in FA, coupled with stable WM metabolites are consistent with remodeling of myelin, perhaps into a more compact structure, during the transition from childhood to adolescence.

\section{Gray matter maturation}

Increasing age was associated with higher FA values in prefrontal, insular, anterior temporal, inferior parietal, and cingulate corti- ces, as well as basal ganglia, thalamus, and cerebellum (Fig. 3). We found little evidence for FA age correlates in posterior temporal, parietal, or occipital cortices. Neuronal components of GM consist primarily of cell bodies, dendritic and axon terminal arbors, and synapses, all with generally less directional orientation than in WM, and GM axon segments are mostly unmyelinated. Most neurons and glia in subcortical GM nuclei, and in some cortical layers, are stellate in shape, further reducing overall directional diffusion of intracellular water. Nevertheless, many neurons and glia in the cortex are oriented perpendicular to the cerebral surface, which can produce a directional coherence in diffusion. Thus, even though DTI studies often exclude FA values $<0.2$ to mask GM, FA and other DTI indices in GM are valid and worth study, especially in larger sample sizes that can overcome the restricted range of DTI indices and lower signal-to-noise in GM (Farrell et al., 2007).

Age-related increases in FA and reductions in ADC and RD in GM, could derive from age-related increases in myelination of WM fibers entering or exiting GM (Lebel et al., 2008), or in the thalamus, from myelinated dendritic spines of medium spiny neurons (Rafols et al., 1989). Alternatively, an age-related increase in cell packing density could produce these findings, because FA correlates strongly with the number of non-myelinated axons in each voxel (Leong et al., 2015); presumably, this would be true not only for increased GM density of neurons and glia, but also for increased GM density of dendritic arbors and synapses in. Although prior morphological imaging studies have shown that 
A
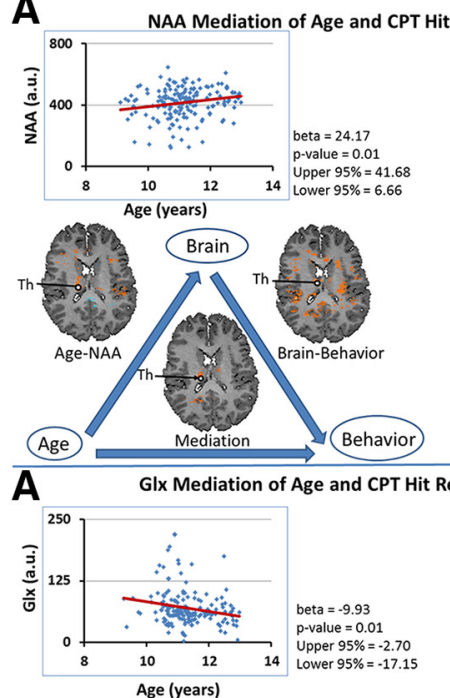

Age (years)

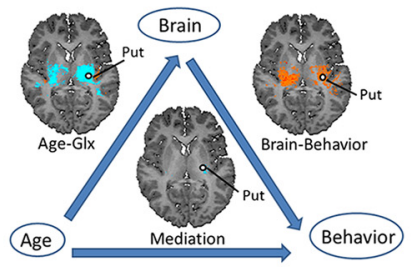

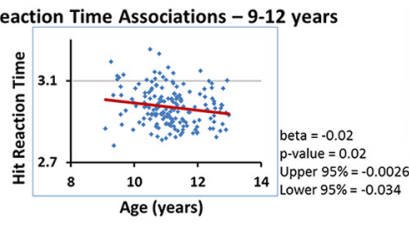
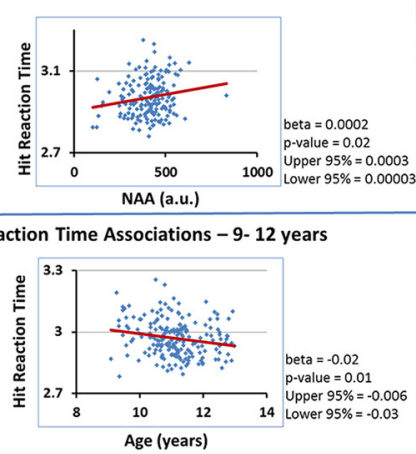

Age (years)
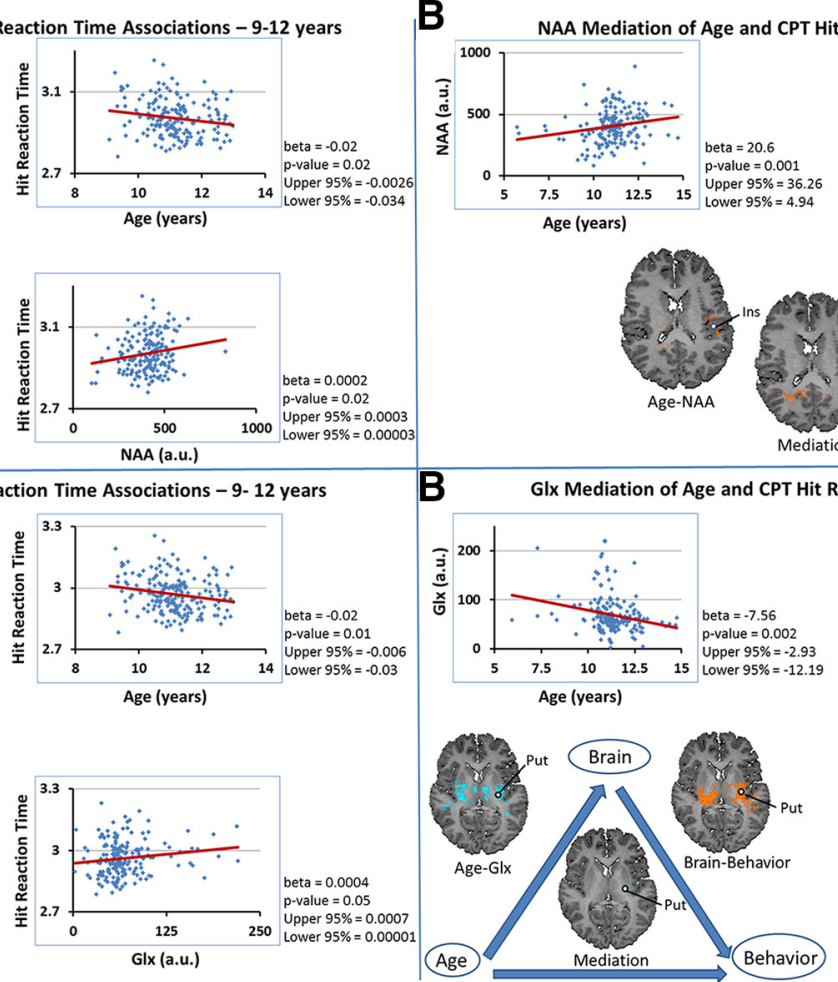

Age (years)
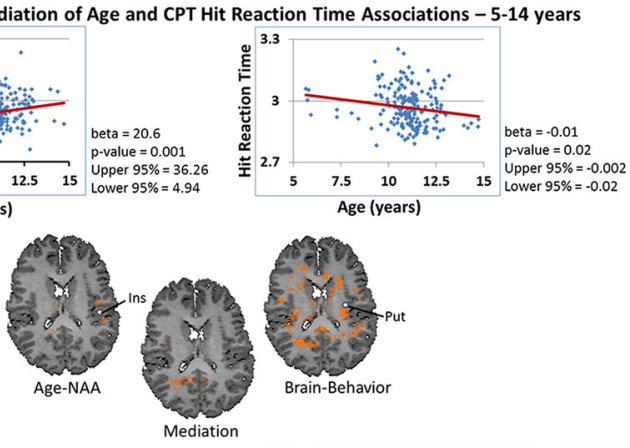

B Glx Mediation of Age and CPT Hit Reaction Time Associations - 5-14 years

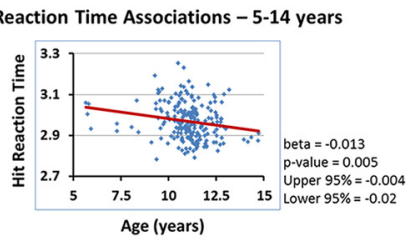

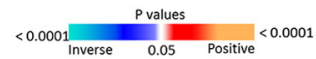

Figure 8. NAA and Glx Mediation with Scatterplots shown here are illustrations of the mediating relationship of NAA or Glx with age and behavioral measures. Representative scatterplots of sampled brain regions are shown for NAA and Glx in children $(\boldsymbol{A})$ 9-12 years and $(\boldsymbol{B})$ 5-14 years, where beta is the degree of change in NAA or Glx per unit change in age. All associations were statistically significant, except for NAA mediation of the association with age with Hit Reaction Time in children ages 5-14. In each panel, the scatterplot on the top left shows the correlation of NAA or Glx with age; that on the top right shows the correlation of behavioral measure with age; and that in the bottom right shows the correlation of behavioral measure with NAA or Glx concentration. Effect size for $\boldsymbol{A}$ (top) was 1.82. Effect size for $\boldsymbol{A}$ (bottom) was -1.48 and for $\boldsymbol{B}$ (bottom) was -1.55 . Ins, Insular cortex; Put, putamen; Th, thalamus.

frontal, temporal, and parietal GM, and GM of the basal ganglia and thalamus, mature throughout late childhood and adolescence (Giedd et al., 1999; Sowell et al., 2003; Shaw et al., 2008; Raznahan et al., 2014; Walhovd et al., 2017), our findings extend these observations to GM microstructure.

We also show, for the first time, variations in GM metabolite concentrations with advancing age. NAA and creatine are key compounds involved in energy metabolism (Chakraborty et al., 2001), and NAA and choline participate in lipid synthesis. Higher NAA concentrations generally reflect increased mitochondrial energy metabolism, increased demand for lipid synthesis, or increased $\mathrm{N}$-acetylaspartylglutamate. Therefore, the observed agerelated GM increases in NAA, creatine, or choline in GM suggest structural or functional growth in these locations, especially dendritic arborization and synaptogenesis, whereas decreases in NAA, creatine, and choline in parieto-occipital cortices suggest dendritic or synaptic pruning.

\section{Microstructure and metabolism mediate age-related improvements in cognitive performance}

FA values in the internal capsule, basal ganglia, and thalamus partially mediated associations of increasing age with shorter reaction times on the CPT and improved performance on Design Copy (Fig. 5). Higher NAA concentrations in the thalamus also partially mediated age-related improvement in CPT reaction times (Fig. 6). Each of these mediation findings had a large effect size, underscoring the robustness of the mediation. These mediation findings suggest that microstructural maturation of the basal ganglia, thalamus, and WM of the internal capsule play central roles in improving performance capacity during the transition from childhood to adolescence. The internal capsule densely interconnects the basal ganglia and thalamus with frontal cortex, which together constitute CSTC circuits. These circuits comprise largely parallel projections from specific cortical regions to the basal ganglia/substantia nigra, then to thalamus, and back to the cortical regions from which the projections originated. They include two separate but interacting pathways: one, the "direct" or excitatory pathway, connects the striatum to the globus pallidus interna/substantia nigra (GPi/SN) complex to generate "go" signals for behavior; the other, an "indirect" pathway, connects the striatum to the globus pallidus externa and subthalamic nucleus, then to the GPi/SN, to generate "stop" signals that serve as a "brake" on the direct pathway and impulses to action (Mink, 1996; Tekin and Cummings, 2002). Balance of activity in these pathways modulates output from the striatum to the thalamus and consequently the output from the thalamus to the cortex. This intricate organization of CSTC circuits positions them to serve as powerful modulating influences on thought, emotion, and behavior. Thus, our findings suggest that maturation of the basal ganglia, thalamus, and WM of the frontal lobes and internal capsule within CSTC circuits supports development of cognitive and self-regulatory capacities during the transition from late childhood into early adolescence.

\section{Limitations}

(1) Inferences about developmental trajectories must be made cautiously in cross-sectional studies such as ours. A prospective longitudinal study with multiple imaging time points is needed to 
conclusively identify developmental trajectories for white and gray matter maturation during this period. It would also permit analyses of within-subject change in imaging measures and cognitive performance, affording more powerful analyses of brainbased mediation of the cognitive and behavioral capacities that emerge in preadolescence. (2) Pubertal status was not assessed concurrently at the time of MRI scanning, precluding an attempt to disentangle the effects of pubertal development and age on our findings. (3) Ours was a sample of urban and racial/ethnic minority participants, and therefore, our findings may not generalize to other populations.

\section{Conclusions}

Our findings of this study suggest a rapid maturation of CSTC pathways during the developmental transition from childhood to adolescence. Maturation of those pathways partially mediates the emergence of greater capacities for self-regulation at a time of increasing academic and social demands. Having defined the normative features of tissue organization and metabolism will permit future study of the ways in which aberrant development of CSTC pathways may support the emergence of behavioral disorders in adolescence that feature impaired self-regulatory control.

\section{References}

Alexander AL, Lee JE, Lazar M, Field AS (2007) Diffusion tensor imaging of the brain. Neurotherapeutics 4:316-329.

Barnea-Goraly N, Menon V, Eckert M, Tamm L, Bammer R, Karchemskiy A, Dant CC, Reiss AL (2005) White matter development during childhood and adolescence: a cross-sectional diffusion tensor imaging study. Cereb Cortex 15:1848-1854.

Beaulieu C (2002) The basis of anisotropic water diffusion in the nervous system: a technical review. NMR Biomed 15:435-455.

Benjamins JA, Smith ME (1984) Metabolism of myelin. In: Myelin (Morell P, ed). Boston: Springer.

Blüml S, Wisnowski JL, Nelson MD Jr, Paquette L, Gilles FH, Kinney HC, Panigrahy A (2013) Metabolic maturation of the human brain from birth through adolescence: insights from in vivo magnetic resonance spectroscopy. Cereb Cortex 23:2944-2955.

Burbridge JB, Magee L, Robb AL (1988) Alternative transformations to handle extreme values of the dependent variable. J Am Stat Assoc 83:123127.

Cercignani M, Inglese M, Pagani E, Comi G, Filippi M (2001) Mean diffusivity and fractional anisotropy histograms of patients with multiple sclerosis. Am J Neuroradiol 22:952-958.

Chakraborty G, Mekala P, Yahya D, Wu G, Ledeen RW (2001) Intraneuronal $N$-acetylaspartate supplies acetyl groups for myelin lipid synthesis: evidence for myelin-associated aspartoacylase. J Neurochem 78:736-745.

Christensen GE, Rabbitt RD, Miller MI (1994) 3D brain mapping using a deformable neuroanatomy. Phys Med Biol 39:609-618.

Colby JB, Van Horn JD, Sowell ER (2011) Quantitative in vivo evidence for broad regional gradients in the timing of white matter maturation during adolescence. Neuroimage 54:25-31.

Colibazzi T, Zhu H, Bansal R, Schultz RT, Wang Z, Peterson BS (2008) Latent volumetric structure of the human brain: exploratory factor analysis and structural equation modeling of gray matter volumes in healthy children and adults. Hum Brain Mapp 29:1302-1312.

Conners CK (1994) The connors continuous performance test (CPT): computer program, version 3.0. North Tonawanda: Multi-Health Systems.

Dong Z, Peterson B (2007) The rapid and automatic combination of proton MRSI data using multi-channel coils without water suppressionl. Magn Reson Imaging 25:1148-1154.

Dubin MJ, Maia TV, Peterson BS (2010) Cognitive control in the service of self-regulation. In: The encyclopedia of behavioral neuroscience (Koob GF, Le Moal M, Thompson RF, eds). Amsterdam: Elsevier.

Farrell JA, Landman BA, Jones CK, Smith SA, Prince JL, Van Zijl PC, Mori S (2007) Effects of SNR on the accuracy and reproducibility of DTIderived fractional anisotropy, mean diffusivity, and principal eigenvector measurements at 1.5T. J Magn Reson Imaging 26:756-767.

Feldman HM, Yeatman JD, Lee ES, Barde LHF, Gaman-Bean S (2010) Diffu- sion tensor imaging: a review for pediatric researchers and clinicians. J Dev Behav Pediatr 31:346-356.

Gibson EM, Purger D, Mount CW, Goldstein AK, Lin GL, Wood LS, al. E, Inema I, Miller SE, Bieri G, Zuchero JB, Barres BA, Woo PJ, Vogel H, Monje M (2014) Neuronal activity promotes oligodendrogenesis and adaptive myelination in the mammalian brain. Science 344:1252304.

Giedd JN, Blumenthal J, Jeffries NO, Castellanos FX, Liu H, Zijdenbos A, Paus T, Evans AC, Rapoport JL (1999) Brain development during childhood and adolescence: a longitudinal MRI study. Nat Neurosci 2:861-863.

Goh S, Dong Z, Zhang Y, DiMauro S, Peterson BS (2014) Mitochondrial dysfunction as a neurobiological subtype of autism spectrum disorder: evidence from brain imaging. JAMA Psychiatry 71:665-671.

Goncalves OF, Carvalho S, Liete J, Fernandes-Goncalves A, Carracedo A, Sampaio A (2016) Cognitive and emotional impairments in obsessivecompulsive disorder: evidence from functional brain alterations. Porto Biomed J 1:92-105.

Hao X, Xu D, Bansal R, Dong Z, Liu J, Wang Z, Kangarlu A, Liu F, Duan Y, Shova S, Gerber AJ, Peterson BS (2013) Multimodal magnetic resonance imaging: the coordinated use of multiple, mutually informative probes to understand brain structure and function. Hum Brain Mapp 34:253-271.

Haselgrove JC, Moore JR (1996) Correction for distortion of echo-planar images used to calculate the apparent diffusion coefficient. Magn Reson Med 36:960-964.

Jáuregui-Lobera I (2011) Neuroimaging in eating disorders. Neuropsychiatr Dis Treat 7:577-584.

Jenkinson M, Bannister P, Brady M, Smith S (2002) Improved optimization for the robust and accurate linear registration and motion correction of brain images. Neuroimage 17:825-841.

Kirk U, Kemp S (2007) Developmental neuropsychological assessment, ed 2 (NEPSY-II). San Antonio, TX: Pearson.

Kessler RM, Hutson PH, Herman BK, Potenza MN (2016) The neurobiologic basis of binge-eating disorder. Neurosci Biobehav Rev 63:223-238.

Lebel C, Walker L, Leemans A, Phillips L, Beaulieu C (2008) Microstructural maturation of the human brain from childhood to adulthood. Neuroimage 40:1044-1055.

Lebon V, Petersen KF, Cline GW, Shen J, Mason GF, Dufour S, Behar KL, Shulman GI, Rothman DL (2002) Astroglial contribution to brain energy metabolism in humans revealed by $13 \mathrm{C}$ nuclear magnetic resonance spectroscopy: elucidation of the dominant pathway for neurotransmitter glutamate repletion and measurement of astrocytic oxidative metabolism. J Neurosci 22:1523-1531.

Leong D, Calabrese E, White LE, Wei P, Chen S, Platt SR, Provenzale JM (2015) Correlation of diffusion tensor imaging parameters in the canine brain. Neuroradiol J 28:12-18.

Leung HC, Skudlarski P, Gatenby JC, Peterson BS, Gore JC (2000) An event-related functional MRI study of the Stroop color word interference task. Cereb Cortex 10:552-560.

MacKinnon DP (2008) Introduction to statistical mediation analysis. New York: Lawrence Erlbaum.

Marsh R, Zhu H, Wang Z, Skudlarski P, Peterson BS (2007) A developmental fMRI study of self-regulatory control in tourette's syndrome. Am J Psychiatry 164:955-966.

Marsh R, Maia TV, Peterson BS (2009a) Functional disturbances within frontostriatal circuits across multiple childhood psychopathologies. Am J Psychiatry 166:664-674.

Marsh R, Steinglass JE, Gerber AJ, Graziano O'Leary K, Wang Z, Murphy D, Walsh BT, Peterson BS (2009b) Deficient activity in the neural systems that mediate self-regulatory control in bulimia nervosa. Arch Gen Psychiatry 66:51-63.

Mink JW (1996) The basal ganglia: focused selection and inhibition of competing motor programs. Prog Neurobiol 50:381-425.

Mukherjee P, Miller JH, Shimony JS, Philip JV, Nehra D, Snyder AZ, Conturo TE, Neil JJ, McKinstry RC (2002) Diffusion-tensor MR imaging of gray and white matter development during normal human brain maturation. Am J Neuroradiol 23:1445-1456.

Nave KA (2010) Myelination and support of axonal integrity by glia. Nature 468:244-252.

Nigg JT (2017) Annual research review: on the relations among selfregulation, self-control, executive functioning, effortful control, cognitive control, impulsivity, risk-taking, and inhibition for developmental psychopathology. J Child Psychol Psychiatry 58:361-383. 
Pavuluri MN, Yang S, Kamineni K, Passarotti AM, Srinivasan G, Harral EM, Sweeney JA, Zhou XJ (2009) Diffusion tensor imaging study of white matter fiber tracts in pediatric bipolar disorder and attention-deficit/hyperactivity disorder. Biol Psychiatry 65:586-593.

Peterson BS, Skudlarski P, Gatenby JC, Zhang H, Anderson AW, Gore JC (1999) An fMRI study of Stroop word-color interference: evidence for cingulate subregions subserving multiple distributed attentional systems. Biol Psychiatry 45:1237-1258.

Peterson BS, Kane MJ, Alexander GM, Lacadie C, Skudlarski P, Leung HC, May J, Gore JC (2002) An event-related functional MRI study comparing interference effects in the simon and Stroop tasks. Brain Res Cogn Brain Res 13:427-440.

Peterson BS, Thomas P, Kane MJ, Scahill L, Zhang H, Bronen R, King RA, Leckman JF, Staib L (2003) Basal ganglia volumes in patients with gilles de la tourette syndrome. Arch Gen Psychiatry 60:415-424.

Power JD, Barnes KA, Snyder AZ, Schlaggar BL, Petersen SE (2012) Spurious but systematic correlations in functional connectivity MRI networks arise from subject motion. Neuroimage 59:2142-2154.

Rae CD (2014) A guide to the metabolic pathways and function of metabolites observed in human brain ${ }^{1} \mathrm{H}$ magnetic resonance spectra. Neurochem Res 39:1-36.

Rafols JA, Cheng HW, McNeill TH (1989) Golgi study of the mouse striatum: age-related dendritic changes in different neuronal populations. J Comp Neurol 279:212-227.

Raine CS (1984) Morphology of myelin and myelination. In: Myelin (Morell P, ed). Boston: Springer.

Ramadan S, Lin A, Stanwell P (2013) Glutamate and glutamine: a review of in vivo MRS in the human brain. NMR Biomed 26:1630-1646.

Raznahan A, Shaw PW, Lerch JP, Clasen LS, Greenstein D, Berman R, Pipitone J, Chakravarty MM, Giedd JN (2014) Longitudinal fourdimensional mapping of subcortical anatomy in human development. Proc Natl Acad Sci U S A 111:1592-1597.

Rubia K (2011) "cool" inferior frontostriatal dysfunction in attention-deficit/hyperactivity disorder versus "hot" ventromedial orbitofrontal-limbic dysfunction in conduct disorder: a review. Biol Psychiatry 69:e69-87.

Shattuck DW, Leahy RM (2002) BrainSuite: an automated cortical surface identification tool. Med Image Anal 6:129-142.

Shaw P, Kabani NJ, Lerch JP, Eckstrand K, Lenroot R, Gogtay N, Greenstein
D, Clasen L, Evans A, Rapoport JL, Giedd JN, Wise SP (2008) Neurodevelopmental trajectories of the human cerebral cortex. J Neurosci 28:3586-3594.

Simmonds DJ, Hallquist MN, Asato M, Luna B (2014) Developmental stages and sex differences of white matter and behavioral development through adolescence: a longitudinal diffusion tensor imaging (DTI) study. Neuroimage 92:356-368.

Smith ME (1968) Lipids and lipid metabolism. Biochim Biophys Acta 164:285-293.

Sowell ER, Peterson BS, Thompson PM, Welcome SE, Henkenius AL, Toga AW (2003) Mapping cortical change across the human life span. Nat Neurosci 6:309-315.

Stoffel W, Hillen H, Giersiefen H (1984) Structural and molecular arrangement of proteolipid protein of central nervous system myelin. Proc Natl Acad Sci U S A 81:5012-5016.

Tekin S, Cummings JL (2002) Frontal-subcortical neuronal circuits and clinical neuropsychiatry: an update. J Psychosom Res 53:647-654.

Uzman BG, Hedley-Whyte ET (1968) Myelin: dynamic or stable? J Gen Physiol 51:8-18.

Viola P, Wells WM (1995) Alignment by maximization of mutual information. In: Proceedings of IEEE International Conference on Computer Vision, pp 16-23, Cambridge, MA: IEEE.

Volkow ND, Koob GF, McLellan AT (2016) Neurobiologic advances from the brain disease model of addiction. N Engl J Med 374:363-371.

Walhovd KB, Fjell AM, Giedd J, Dale AM, Brown TT (2017) Through thick and thin: a need to reconcile contradictory results on trajectories in human cortical development. Cereb Cortex 27:1472-1481.

Yakovlev PL, Lecours AR (1967) The myelogenetic cycles of regional maturation of the brain. In: Resional development of the brain in early life (Minkowski A, ed), pp 3-70. Oxford: Blackwell.

Yeung MS, Zdunek S, Bergmann O, Bernard S, Salehpour M, Alkass K, Perl S, Tisdale J, Possnert G, Brundin L, Druid H, Frisén J (2014) Dynamics of oligodendrocyte generation and myelination in the human brain. Cell 159:766-774.

Young KM, Psachoulia K, Tripathi RB, Dunn SJ, Cossell L, Attwell D, Tohyama K, Richardson WD (2013) Oligodendrocyte dynamics in the healthy adult CNS: evidence for myelin remodeling. Neuron 77 : 873-885. 